\title{
1 Effects of magnetic nanoparticles on mixing in droplet-based 2 microfluidics
}

4 Mohammad Amin Maleki, ${ }^{1}$ M. Soltani, ${ }^{1,2,3,4, a)}$ Navid Kashaninejad, ${ }^{5}$ Nam-Trung Nguyen,, ,b)

$6 \quad{ }^{1}$ Department of Mechanical Engineering, K. N. Toosi University of Technology, Tehran 19697, Iran

$7 \quad{ }^{2}$ Department of Electrical and Computer Engineering, University of Waterloo, ON N2L 3G1, Canada

$8{ }^{3}$ Centre for Biotechnology and Bioengineering (CBB), University of Waterloo, Waterloo, ON N2L 3G1, Canada

$9 \quad{ }^{4}$ Cancer Biology Research Centre, Cancer Institute of Iran, Tehran University of Medical Sciences, Tehran 13145-

10158, Iran

$11{ }^{5}$ Queensland Micro- and Nanotechnology Centre and ${ }^{\ddagger}$ School of Natural Science, Griffith University, Nathan

12 Campus, 170 Kessels Road, Brisbane, QLD 4111, Australia

13 Published Online: 05 March 2019 Accepted: February 2019

14 Physics of Fluids 31, 032001 (2019); https://doi.org/10.1063/1.5086867

\section{Abstract} as that in a microchannel is of great importance for lab-on-a-chip applications. Various techniques for mixing enhancement in microfluidics have been extensively reported in the literature. One of these techniques is the mixing enhancement with magnetofluidics using ferrofluid, a liquid with dispersed magnetic nanoparticles. However, a systematic study exploring the mixing process of ferrofluid and its influencing parameters is lacking. The present study numerically examines the effect of key parameters including magnetic field,

\footnotetext{
a) Author to whom correspondence should be addressed: madsoltani@uwaterloo.ca

b) Author to whom correspondence should be addressed: nam-trung.nguyen@griffith.edu.au (N.-T. Nguyen).
} 
mean velocity and size of a microdroplet on the mixing process. A microfluidic double Tjunction with droplets in merging regime is considered. One of the dispersed phases is a ferrofluid containing paramagnetic nanoparticles, while the other carried neutral species. Under an applied magnetic field, the ferrofluid experiences a magnetic force that in turn induces a secondary bulk flow called magnetoconvection. The combination of the induced magnetoconvection and shear-driven circulating flow within a moving droplet improves the mixing efficiency remarkably. Mixing enhancement is maximized for a specific ratio between the magnetic force to the shear force. The dominance of either force would deteriorate the mixing performance. On the other hand, using a magnetic force and a shear force with comparable order of magnitude leads to effective manipulation of vortices inside the droplet, and subsequently causes an optimized particles distribution over the entire droplet. Furthermore, the smaller the droplets are the better is mixing.

\section{I. INTRODUCTION}

Microfluidic technology holds a great potential for biomedical applications. This technology

37 allows for implementing laboratory operations using a small volume of reagents in a short time. ${ }^{1}$ Droplet-based microfluidics is an important branch of microfluidics, where discrete droplets are handled within an immiscible carrier fluid. Because of the encapsulation and protection of the droplets by the surrounding immiscible fluid, such systems can function as a sample test tube or a microreactor in small scale. ${ }^{2}$ Droplet-based microfluidics has several advantages over continuous-

42 flow microfluidics, including the elimination of cross-contamination of the analytes, ultra-high-

43 throughput screening process, a significant reduction in consumption of reagents due to the small

44 volume of the droplets, the capability of high throughput generation, manipulation, and processing 45 of ultra-small microreactors. ${ }^{3}$ These advantages lead to the growing use of droplet-based 
46 microfluidics in applications such as studying cell mechanical behavior and tissue aspiration, ${ }^{4}$ drug

47 screening and discovery, ${ }^{5}$ chemical and biochemical synthesis, ${ }^{6}$ DNA and genomic studies, ${ }^{7}$ cell

48 culture, $^{8}$ and diagnostics. ${ }^{9}$

49 However, high-throughput combinatorial assays in droplet-based microfluidics require the 50 implementation of multiple steps such as compartmentalization, manipulation and measurement. ${ }^{\mathbf{1 0}}$

51 Most useful operations are droplet generation, ${ }^{11}$ transport, ${ }^{12}$ sorting, ${ }^{13}$ separation, ${ }^{14}$ merging, ${ }^{15}$

52 trapping ${ }^{16}$ and mixing the droplet contents. ${ }^{4,}{ }^{17}$ Diverse mechanisms have been employed for 53 droplet handling such as electrowetting, ${ }^{18}$ dielectrowetting, ${ }^{15}$ magnetofluidic, ${ }^{19}$ photoactuation, ${ }^{20}$ 54 hydrodynamic, ${ }^{10}$ surface acoustic waves, ${ }^{2}$ chemical gradient,${ }^{21}$ surface morphological gradients ${ }^{22}$ 55 and temperature gradient. ${ }^{23}$

56 Mixing is one of the basic microfluidic operations that plays a crucial role in the above 57 applications. Nevertheless, obtaining uniform mixing in microfluidic devices in a short time has 58 been challenging because of the low Reynolds number of the resulting laminar flow in 59 microchannels. ${ }^{24}$ Due to the more rapid mixing process of microdroplet in a multi-phase system 60 compared to that in a single-phase system and their advantage in elimination the dispersion effect, 61 microdroplets are frequently used to enhance mixing in microfluidics. ${ }^{25}$ Traditionally, mixing 62 inside droplets is enhanced using chaotic advection. In a straight channel, devoloping a chaotic 63 flow in droplets is challenging due to the symmetric internal flow recirculation.

64 Therefore, various experimental and numerical studies have been conducted to improve the mixing 65 performance of droplet-based microfluidics, using either passive or active techniques. ${ }^{17,26,27}$ In 66 passive mixers, chaotic advection inside the droplet is introduced by passing the droplet through 67 serpentine, sinusoidal and meandering microchannels. A droplet traveling in a curved 68 microchannel establishes asymmetric recirculation, which allows for convective mass transfer 
69 enhancement in both axial and radial directions. ${ }^{28}$ While passive micromixers include the intricate

70 structure of microchannel or long mixing length to achieve the desired mixing output, active

71 mixers utilize external energy to induce flow disturbance to enhance mixing. ${ }^{29}$ Exploiting magnetic

72 force is a convenient technique because of advantages such as convenient bio-functionalizing of

73 magnetic particles, remote control and straightforward design. ${ }^{24,}{ }^{26}$ Combining magnetism and

74 fluid flow in the microscale for actuation and sensing applications led to the field of micro 75 magnetofluidics. ${ }^{30}$

76 Several studies devoted to mixing within microdroplets using magnetic particles. Part of these

77 studies investigated droplets merging and mixing using magnetic nano/microparticles on digital

78 micro magnetofluidic platforms. ${ }^{31}$ Even though digital platforms provide the possibility of precise

79 control and manipulation of a single droplet, their low throughput may prevent them from

80 applications such as drug screening, where a large number of droplets are manipulated

81 simultaneously. Magnetic stirring inside a droplet is an alternative strategy for mixing

82 enhancement. Magnetic polymer beads, ${ }^{32}$ polymer structures containing magnetic nanoparticles, ${ }^{26}$

83 and magnetic bars have been used for stirring inside droplets. In this technique, the dipole-dipole

84 interaction between magnetic particles in the presence of a magnetic field causes the formation of

85 a particle chain forming the stirrer. These structures are actuated by an external rotational magnetic

86 field leading to mixing inside a droplet. Sedimentation of large magnetic stirrers and the need for

87 a very strong magnetic field for smaller stirrers are the main problems of this approach. ${ }^{26}$ Despite

88 the above experimental works, there is a lack of detailed study that numerically investigates

89 magnetofluidic mixing process of ferrofluid and its efficiency.

90 Herein, we numerically evaluate the influence of the magnetic field and inhomogeneous magnetic

91 fluid on the mixing process within a microdroplet. The concentration gradient of magnetic particles 
92 inside the droplet lead to magnetic moment gradient and consequently a magnetic bulk force, even

93 in a uniform magnetic field. The induced magnetic force contributes to a secondary flow inside

94 the droplet when it is passing through a straight channel. This secondary flow makes the internal

95 circulations asymmetric, leading to chaotic advection and significant mixing enhancement. We

96 first compare droplet mixing with and without a magnetic field, where we consider ferrofluid as

97 one of the dispersed phases. We will show that the magnetic field can be very beneficial for

98 significantly enhancing the mixing process inside the droplet.

99

100

101

102

103

104

105

106

$107 \quad \mathbf{F}_{s}=\nabla \cdot\left(\sigma\left(\mathbf{I}-\left(\mathbf{n n}^{T}\right)\right) \delta\right)$

\section{MATHEMATICAL MODELING AND IMPLEMENTATION}

\section{A. Governing equations and boundary conditions}

Incompressible Navier-Stokes equation is employed to describe the flow dynamics: ${ }^{33}$

$$
\rho \frac{\partial \mathbf{u}}{\partial t}+\rho(\mathbf{u} \cdot \nabla) \mathbf{u}=-\nabla p+\eta \nabla^{2} \mathbf{u}+\mathbf{F}_{s}+\mathbf{F}_{m}
$$

In this equation, $\rho$ represents the density of the fluid, $p$ the pressure, $\eta$ the dynamic viscosity and $\mathbf{F}_{s}$ the force caused by surface tension and $\mathbf{F}_{m}$ the magnetic volume force. Surface tension body force $\mathbf{F}_{s}$ is calculated as: ${ }^{34}$

where $\mathrm{n}$ is a unit vector that is normal to the interface, $\sigma$ is the interfacial tension between the two phases and $\delta$ is delta function with zero value in all domain except fluids interface. This 110 function is expressed as follow:

$$
\delta=6|\nabla \varphi||\varphi(1-\varphi)|
$$


112 Where $\varphi$ represents the level-set function that is used to model the interaction of immiscible fluids

113 and govern their interface movement. In the level-set method, the level-set function $\varphi$ determines

114 the distinct phases and portrays a smooth transition across the interface from one to zero by a

115 Heaviside function, ${ }^{34}$ the conservative level set equation is defined as follow:

$116 \quad \frac{\partial \varphi}{\partial t}+\mathbf{u} \cdot \nabla \varphi=\gamma \nabla \cdot\left(\varepsilon \nabla \varphi-\varphi(1-\varphi) \frac{\nabla \varphi}{|\nabla \varphi|}\right)$

117 where $\gamma$ represent the reinitialization parameter and the $\varepsilon$ denotes interface thickness where level

118 set function vary from 0 to 1 (for core $\varphi=1$ and for carrier $\varphi=0$ ). Reinitialization is a necessary

119 step to maintain the signed distance property and control the numerical stabilization.

120 The following convective/diffusive equation describes the non-dimensional mass transport

121 throughout the fluidic domain; this equation is used to model the distribution of magnetic particles

122 in the computational domain: ${ }^{35}$

$123 \quad \frac{\partial c}{\partial t}+\mathbf{u}_{p} \cdot \nabla c=\nabla \cdot(D \nabla c)$

124 where $c$ represents dimensionless concentration, $D$ denotes diffusivity coefficient and $\mathbf{u}_{p}$

125 denotes the velocity of the particles. The diffusive behavior of the magnetic particles is affected 126 by magnetic force. This effect is shown by an additional velocity component that is given by Stokes 127 drag law:

$128 \mathbf{u}_{p}=\mathbf{u}+\mathbf{u}_{m a g}=\mathbf{u}+\mathbf{F}_{m a g} / 6 \pi \eta r_{p}$ 
129 where $r_{p}$ shows magnetic particle radius and $\mathbf{F}_{m a g}$ is the magnetic force on each particle which is 130 given by:

$131 \quad \mathbf{F}_{m a g}=\frac{1}{2} V_{p} \mu_{0} \nabla \chi \mathbf{H}^{2}$

132 where $V_{p}$ is the volume of any single magnetic particle, $\mu_{0}$ is the permeability of the vacuum space,

$133 \chi$ is the local susceptibility of the ferrofluid and $\mathbf{H}$ is the magnetic field strength.

134 Considering the distinct solubility of species (i) in each phase, there is a concentration jump across

135 the fluid interface. The interfacial discontinuity of concentration can be expressed as follow: ${ }^{36}$

$136 \quad c_{c}=K c_{d}$

137 where $K$ is the distribution coefficient. The indices $c$ and $d$ correspond to the continuous and 138 dispersed phase, respectively. In addition, flux continuity condition is established at the interface:

$139 \quad D_{d} \frac{\partial c_{d}}{\partial \mathbf{n}}=D_{c} \frac{\partial c_{c}}{\partial \mathbf{n}}$

140 Because of the moving interface, it is difficult to apply the boundary condition to this area. Keniga

141 et al. ${ }^{34}$ suggested a new method to address this problem. This method incorporates interfacial

142 boundary conditions to the mass transport equation so that these conditions are satisfied only in

143 the proximity of the immiscible fluids interface. Considering the above boundary conditions, new

144 source terms are added to the mass transport equation. Accordingly, the following equations are 145 obtained and solved for all the fluidic domains: 
$146 \quad \frac{\partial c_{d}}{\partial t}+\mathbf{u}_{p} \cdot \nabla c_{d}=\nabla \cdot\left(D \nabla c_{d}\right)+\alpha_{1}\left(D_{c} \frac{\partial c_{c}}{\partial \mathbf{n}}-D_{d} \frac{\partial c_{d}}{\partial \mathbf{n}}\right)$

$147 \quad \frac{\partial c_{c}}{\partial t}+\mathbf{u}_{p} \cdot \nabla c_{c}=\nabla \cdot\left(D \nabla c_{c}\right)+\alpha_{2}\left(c_{c}-\frac{c_{d}}{K}\right)$

148 To fulfill the boundary condition at the interface, the values of $\alpha_{1}$ and $\alpha_{2}$ should be considered as

149 large enough at the interface and zero in other places. For this purpose, we have implemented a

150 multiple of Dirac distribution ${ }^{37}$ of the level set $\varphi$ variable as $\alpha_{1}$, as well as, $\alpha_{2}$. Consequently, $\alpha_{1}$

151 and $\alpha_{2}$ are defined according to the following relation:

$152 \alpha_{1}=\alpha_{2}=K$ fldc $2 h s((\varphi-0.5), q)= \begin{cases}0 & \text { if }|\varphi-0.5|>q \\ \frac{K}{2 q}\left[1+\cos \left(\frac{\pi(\varphi-0.5)}{q}\right)\right] & \text { if }|\varphi-0.5| \leq q\end{cases}$

153 Doing so, we have applied the maximum value to $\alpha_{1}$ and $\alpha_{2}$ at the interface were $\varphi$ and zero

154 value at $|\varphi-0.5|>q$. Given that $\varphi$ varies from zero to one at the interface, $q$ can take any value

155 from between 0 and 0.5 .

156 Maxwell equations were employed to calculate induced magnetic force on magnetic particles

157 within the ferrofluid. For non-conducting media, Maxwell equations are expressed as follow: ${ }^{38}$

$158 \nabla \times \mathbf{H}=\mathbf{0}$

$159 \nabla \cdot \mathbf{B}=\mathbf{0}$

160 where $\mathbf{H}$ is the magnetic field strength and $\mathbf{B}$ is the magnetic flux density. The relationship

161 between the fields $\mathbf{H}$ and $\mathbf{B}$ for both magnetic and nonmagnetic media is: 
$\mathbf{B}= \begin{cases}\mu_{0}(\mathbf{M}+\mathbf{H}), & \text { if } \Omega_{f} \\ \mu_{0} \mathbf{H}, & \text { if } \Omega_{n}\end{cases}$

163 where $\mu_{0}$ is the permeability constant of the vacuum space with the value of $4 \pi \times 10^{-7} N / A^{2}$ and 164 M denotes the magnetization of the ferrofluid. Langevin's magnetization function describes the 165 magnetization behavior of the ferrofluid. According to this relation the magnetic nanoparticles 166 have linear magnetization at low magnetic fields, which we have considered in this work for 167 modeling the magnetization: ${ }^{39}$

$168 \mathbf{M}(\mathbf{H})=\chi_{f f} \mathbf{H}=M_{s}(\cot (\gamma H)-1 / \gamma H) \mathbf{H} / H$

169 where $M_{s}$ shows saturation magnetization, $\gamma$ is the Langevin parameter $\left(\gamma=3 \chi_{0} / M_{s}, \chi_{0}\right.$ is the 170 initial susceptibility), $H=|\mathbf{H}|$ and $\chi_{f f}$ is magnetic susceptibility of the ferrofluid which is 171 defined as:

$172 \chi_{f f}=\left(M_{s} / H\right)(\cot (\gamma H)-1 / \gamma H)$

173 The flux density inside the ferrofluid can be expressed as $\mathbf{B}=\mu_{0}(1+\chi) \mathbf{H}=\mu \mathbf{H}$. Introducing the 174 magnetic scalar potential $\psi$ that takes the form of $\mathbf{H}=-\nabla \psi$ yielding: ${ }^{35,40}$

$175 \nabla \cdot((1+\chi) \nabla \psi)=0$

176 The concentration gradient of the magnetic nanoparticles, results in a magnetic permeability 177 gradient across the domain. Considering the magnetic potential equation (18), the variation of the 178 magnetic permeability inside the domain results in a gradient of the magnetic potential and the 179 local magnetic field within the domain, while the magnetic field far from permeability variations 
180 (near surrounding media boundaries) remains uniform. The magnetic susceptibility on the entire 181 computational domain is obtained based on the harmonic mean: ${ }^{40}$

$182 \frac{1}{1+\chi}=\frac{1-\varphi}{1+\chi_{c}}+\frac{\varphi}{1+\chi_{d}}$

183 where $\chi_{c}$ and $\chi_{d}$ indicate the susceptibility of the continuous and dispersed phases, respectively.

184 Considering a very small solubility of magnetic particles in the continuous phase and the very 185 large value of $K$, the susceptibility of this phase is approximated as zero. ${ }^{41}$ Furthermore, because 186 of the existence of two miscible phases and the concentration gradient inside the dispersed phase, 187 the susceptibility of the dispersed phase was chosen to be a function of the magnetic particles 188 concentration $\left(\chi_{d}(c)=c \chi_{f f}, \chi_{f f}\right.$ is the magnetic susceptibility of ferrofluid with $\left.c=1\right)$. The magnetic 189 force in a uniform magnetic field and for a linearly magnetizable medium is expressed as follow: ${ }^{38}$

$190 \quad \mathbf{F}_{m}=-\frac{1}{2} \mu_{0} C_{v}|\mathbf{H}|^{2} \nabla \chi$,

191 where $C_{v}$ is the volume concentration of the magnetic particles $\left(C_{v}=c C_{v 0}, C_{v 0}\right.$ is the initial 192 volume concentration). Also, approximating zero value for the susceptibility of the continuous 193 phase, the gradient of the susceptibility is calculated as $\frac{\partial \chi}{\partial \varphi} \nabla \varphi+\frac{\partial \chi}{\partial \chi_{d}} \nabla \chi_{d}$. The first term expresses 194 the variation of the susceptibility on the droplet interface, and the second one demonstrates the 195 variation of the susceptibility inside the droplet due to the magnetic particles concentration 196 gradient in the associated regions. According to equation (9) for a uniform magnetic field, the 197 magnetic force acts on the areas where the susceptibility gradient exists. In our model, the 198 concentration gradient inside the droplet and the permeability discontinuity across the interface 199 give rise to susceptibility gradient and subsequently magnetic force in these domains. The 
200 magnetic potential at the surrounding media boundaries was determined as the boundary condition

201 for solving equation (18) and obtaining the magnetic potential within the entire domain. The

202 magnetic potential on the surrounding media boundaries is calculated as follows: ${ }^{35,41}$

$203 \quad \mathbf{H} \cdot \mathbf{n}=-\frac{\partial \psi}{\partial \mathbf{n}}, \forall \mathbf{x} \in \Omega$

204 The variation of the density and viscosity is taken into account by expressing them as functions

205 of concentration and phase field:

$206 \rho_{\mathrm{d}}=c \rho_{\mathrm{ff}}+(1-c) \rho_{\mathrm{m}}$

$207 \quad v_{\mathrm{d}}=v_{\mathrm{ff}} e^{R(1-c)}$

$208 \rho=\varphi \rho_{\mathrm{d}}+(1-\varphi) \rho_{\mathrm{c}}$

$209 v=\varphi v_{\mathrm{d}}+(1-\varphi) v_{\mathrm{c}}$

210 Where $\rho$ represents the density, $v$ the viscosity and indices $\mathrm{c}, \mathrm{d}, \mathrm{m}$ and $\mathrm{ff}$ stands for continuous

211 phase, disperse phase, deionized water-glycerol mixture and ferrofluid respectively. Furthermore,

$212 R$ is expressed as $R=\ln \left(v_{\mathrm{m}} / v_{\mathrm{ff}}\right)$

213 To evaluate the mixing performance, the mixing index parameter is employed which is expressed 214 as: $^{42}$

$215 \quad M_{i}=1-\frac{\int_{A}\left|c_{i}-c_{d}\right| d A}{\int_{A}\left|c_{0}-c_{d}\right| d A}$

216 where $c_{i}$ is the normalized concentration of species based on the inlet concentration, $c_{d}$ is the 217 average normalized concentration of species in the droplet, $c_{0}$ is the initial normalized 
218 concentration and the $\mathrm{d} A$ is the infinitesimal area of the droplet.".$c_{0}$ can change from 0 to 1

219 corresponding to fully unmixed and mixed solutions, respectively.

221 B. Numerical implementation

222 The presented models are implemented with the Finite Element Method (FEM) based software

223 COMSOL, which allows for solving partial differential equations in a fully coupled approach. The

224 implicit fifth order backward differential formula (BDF) is employed for temporal discretization,

225 which is integrated with the spatial discretized partial differential equations. ${ }^{43}$ Then, the set of

226 obtained nonlinear equations in each time step is linearized by Newton method. ${ }^{44}$ We used the

227 Segregated approach, which treats each physics sequentially (Fig. 1), to solve the multiphysics

228 finite elements. In this approach, the results of the previously solved physics are used to evaluate

229 the loads and material properties for the next physics. Thus, first, the fluid flow solution with

230 variables such as velocity field, pressure, level set function and Reciprocal initial interface distance

231 was evaluated in each time step using guesting an initial value for material distribution and

232 variables. Subsequently, the concentration distribution was evaluated with the solution from fluid

233 flow and an initial value for the magnetic field. Finally, with the fluid flow and the mass transport

234 solution that had been just computed, the magnetic field was solved. PARDISO solver was utilized

235 for direct solving of the linearized equations. Besides accuracy, this solver is well known to be 236 memory efficient and a robust tool for parallel computations. Also, because of the fast factorization

237 process in this solver, the overall required solution time is reduced compared to the other direct

238 solvers. ${ }^{45}$ Moreover, to have an accurate approximation of the curvature and to preserve the level

239 set function and the shape of the interface, a reinitialization step was used that requires the equation

240 to be solved for a few pseudo time steps for each actual time step. For Navier-Stokes and mass 
241 conservation equations, $\mathrm{P} 2+\mathrm{P} 1$ elements were set which provides the second order basis for 242 velocity field and the first order basis for pressure. Level set and Maxwell equations were also 243 solved via the second order discretization. Furthermore, considering high Peclet number, linear 244 elements were utilized for mass transport physic. ${ }^{46}$

245 Crosswind diffusion and stream diffusion stabilizers were applied to mass transport, as well as, 246 two-phase flow physics to achieve more robust and faster computational performance. These 247 artificial diffusion terms eliminate nonphysical local oscillations and capture discontinuities, 248 however using coarse mesh or having considerable heterogeneity in mesh distribution gives rise 249 to a deflection from the exact solution. To minimize this deviation, highly dense and smoothed 250 mesh (with a maximum size of 6 micrometers) was employed in the channel geometry of our 251 model ${ }^{47}$ For capturing the interface movement, COMSOL implements a conservative level set 252 that has been developed by Olsson and Kreiss. ${ }^{34}$ The main advantage of the conservative level set 253 method compared to the standard level set method is preserving of the original area even in the 254 highly curved evolving surfaces. ${ }^{48}$ 


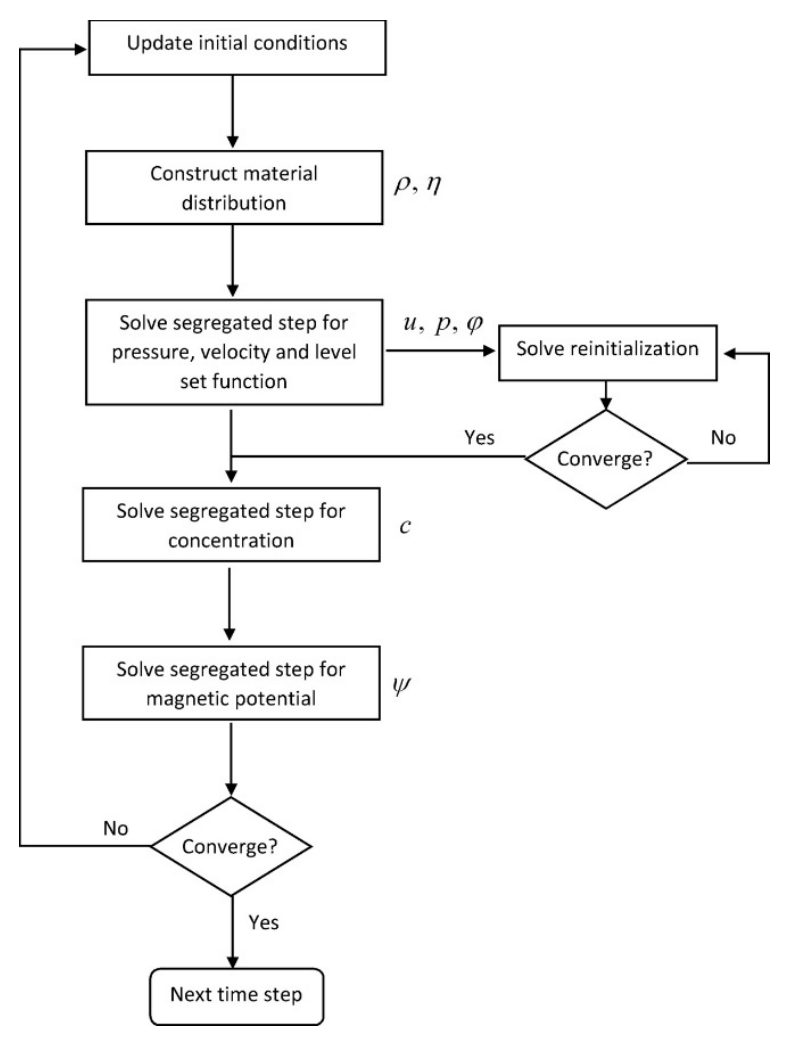

Fig. 1 Flowchart showing the numerical procedure

\section{RESULTS AND DISCUSSION}

\section{A. Validation}

\section{Effect of magnetic field on the formation of ferrofluid droplets}

262 Our model consists of a magnetic field effect on ferrofluid in both miscible and immiscible phases.

263 Thus, the numerical model is first examined for the magnetic effect on ferrofluid droplets. To this 264 aim, we validated the three-dimensional (3D) formation of ferrofluid droplets exposed to a uniform 265 magnetic field reported by Liu et al. ${ }^{40}$ They explored the droplet formation process under magnetic 266 field influence in a flow focusing configuration, both numerically and experimentally. Based on

267 these studies, the magnetic force was acting on the droplet interface due to the permeability 268 discontinuity. Consequently, in the presence of a magnetic field parallel to the ferrofluid flow 
269 direction, the additional magnetic force pulled the ferrofluid forward, elongated its tip as well as,

270 its throat and delayed droplet formation. Thereby, the droplets formed under the magnetic field

271 have a larger volume compared to those formed in the absence of the magnetic field. The authors

272 considered the Bond number and magnetic susceptibility as indicators of magnetic field influence

273 on droplet formation, and illustrated the size and shape dependence of the droplet to these factors.

274 Figs. 2 and 3 show the droplet generation process for two cases without and with a magnetic field,

275 respectively. The results illustrate a good agreement between our results and Liu et al. study. ${ }^{40}$ For

276 the first and the second cases (without and with the magnetic field), the dimensionless volume of

277 the generated droplets in our work are approximately 0.71 and 1.16, and that of Liu et al. study ${ }^{40}$

278 are approximately 0.83 and 1.25 , respectively.

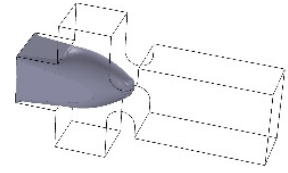

$\mathrm{l}=0 \mathrm{~ms}$

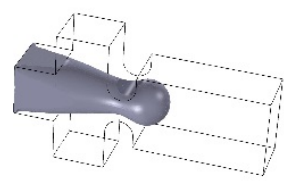

$\mathrm{l}=116 \mathrm{~ms}$

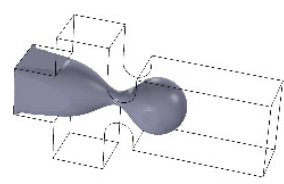

$\mathrm{t}=173 \mathrm{~ms}$

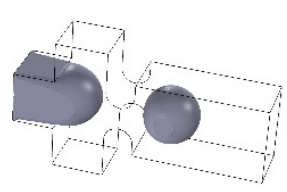

$t=209$

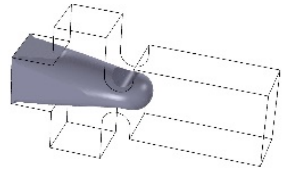

$\mathrm{t}=47 \mathrm{~ms}$

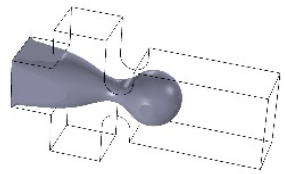

$\mathrm{t}=166 \mathrm{~ms}$

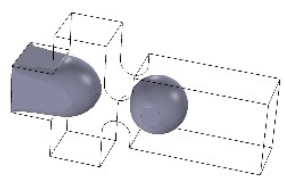

$\mathrm{t}=187 \mathrm{~ms}$

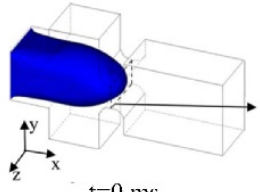

$\mathrm{t}=0 \mathrm{~ms}$

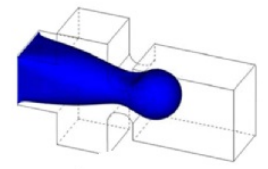

$\mathrm{t}=116 \mathrm{~ms}$

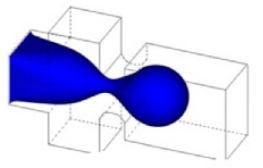

$\mathrm{t}=173 \mathrm{~ms}$

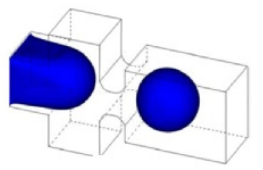

$\mathrm{t}=209 \mathrm{~ms}$

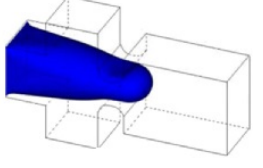

$\mathrm{t}=47 \mathrm{~ms}$

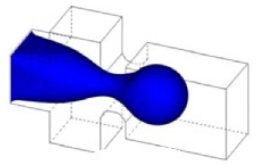

$\mathrm{t}=166 \mathrm{~ms}$

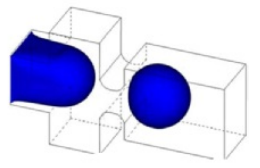

$\mathrm{t}=187 \mathrm{~ms}$

280 FIG. 2 Ferrofluid droplet formation without the magnetic field ( $\mathrm{Ca}=2.22 \times 10^{-3}$

$281, \operatorname{Re}=2.78 \times 10^{-4} Q_{2}=10 \mu l / h, Q_{1}=5 \mu l / h, B_{m}=0, \chi_{0}=8$ ), (a) present results (b) Liu et al. 
282 study $^{40}$. Reproduced from J. Liu, Y. F. Yap, and N.-T. Nguyen, "Numerical study of the 283 formation process of ferrofluid droplets," Phys. Fluids, 23, 072008 (2011), with the permission 284 of AIP Publishing.

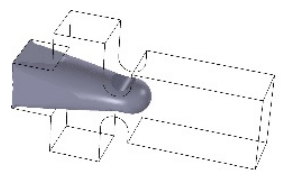

$\mathrm{t}=43 \mathrm{~ms}$

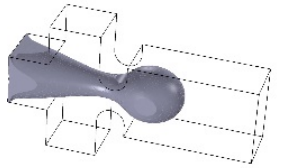

$\mathrm{t}=223 \mathrm{~ms}$

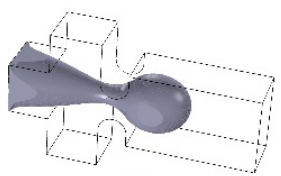

$\mathrm{t}=266 \mathrm{~ms}$

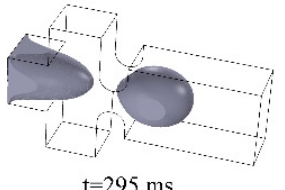

$\mathrm{t}=295 \mathrm{~ms}$

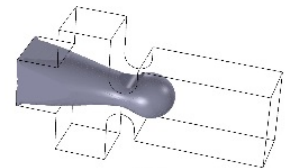

$\mathrm{t}=108 \mathrm{~ms}$

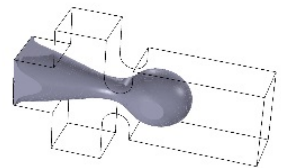

$\mathrm{t}=244 \mathrm{~ms}$

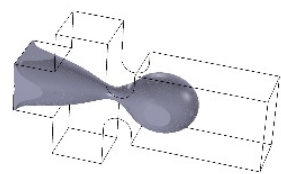

$\mathrm{t}=288 \mathrm{~ms}$

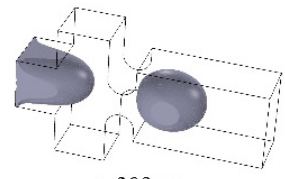

$\mathrm{t}=309 \mathrm{~ms}$

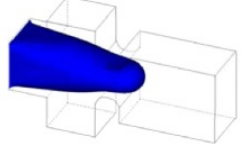

$\mathrm{t}=43 \mathrm{~ms}$

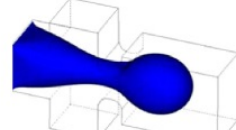

$\mathrm{t}=223 \mathrm{~ms}$

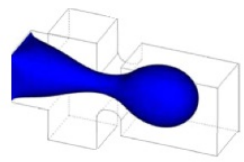

$t=266 \mathrm{~ms}$

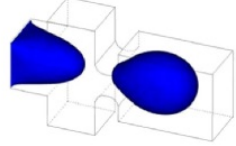

$\mathrm{t}=295 \mathrm{~ms}$

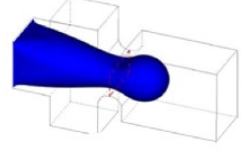

$\mathrm{t}=108 \mathrm{~ms}$

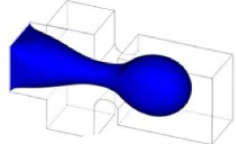

$\mathrm{t}=244.6 \mathrm{~ms}$

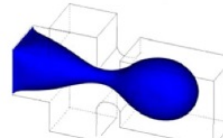

$\mathrm{l}=288 \mathrm{~ms}$

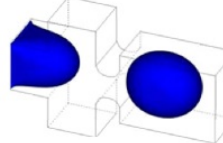

$\mathrm{t}=309.4 \mathrm{~ms}$

(a)

(b)

286

287

288

289

290

291

292

293

294

295

FIG. 3 Ferrofluid droplet formation with the uniform magnetic field ( $\mathrm{Ca}=2.22 \times 10^{-3}$, $\operatorname{Re}=2.78 \times 10^{-4}, Q_{2}=10 \mu l / h, Q_{1}=5 \mu l / h, B_{m}=0, \chi_{0}=8$ ), (a) present results (b) Liu et al. study $^{40}$. Reproduced from J. Liu, Y. F. Yap, and N.-T. Nguyen, "Numerical study of the formation process of ferrofluid droplets," Phys. Fluids, 23, 072008 (2011), with the permission of AIP Publishing.

\section{Magnetic field effect on bulk flow mixing improvement}

After validating the numerical model for magnetic field effect on the immiscible two-phase system

the numerical model is tested for magnetofluidic mixing of two miscible phases. We tried to replicate the Zhu and Nguyen work ${ }^{35}$ that experimentally and numerically investigated the mixing of ferrofluid inside a circular chamber. In this study, high Peclet number and a negligible diffusive 
296 mixing led to a sharp concentration gradient across the interface, which induced a large 297 susceptibility gradient and consequently instability when a uniform magnetic field was applied. 298 The mixing was studied for both ferrofluid-core and ferrofluid-cladding cases. Mixing 299 enhancement occurred in two regimes: (i) low magnetic field regime, where magnetophoresis of 300 the magnetic nanoparticles improved diffusive mixing, and (ii) strong magnetic field regime, 301 which generates a bulk secondary flow and accelerates mixing due to magnetoconvection. 302 Moreover, the effect of parameters such as flow rate ratio, flow rate and viscosity on mixing 303 efficiency are investigated. In the numerical section of the Zhu and Nguyen study ${ }^{35}$ variation of 304 mixing efficiency over a range of magnetic field strength was verified. Water-based ferrofluid and 305 a mixture of DI water and glycerol were used as miscible fluids. Flow rates of $0.5 \mathrm{ml} / \mathrm{h}$ were 306 considered for both fluids. Also, the viscosity ratio of diamagnetic to ferrofluid was fixed at 0.5. 307 Subsequently, we compared our results with those obtained by Zhu and Nguyen study ${ }^{35}$ in Fig. 4. 308 Our results show an acceptable agreement with Zhu and Nguyen study. According to our results, 309 the mixing index for ferrofluid core is approximately 0.75 and that for the ferrofluid cladding 310 isapproximately 0.86 . Consistently, Zhu and Nguyen obtained the values of 0.74 and 0.9 for 311 ferrofluid core and the ferrofluid cladding, respectively. 


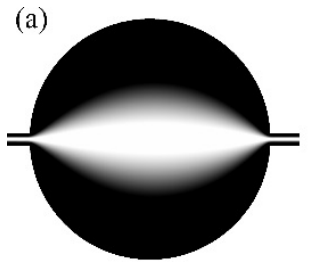

(i)

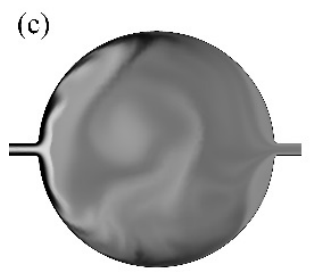

(i)

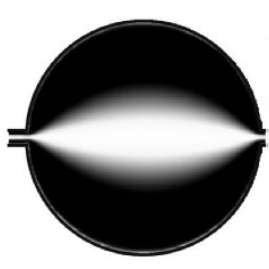

(ii)

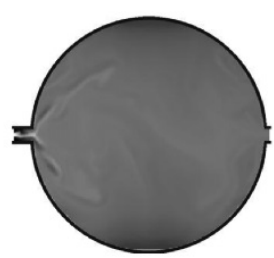

(ii)

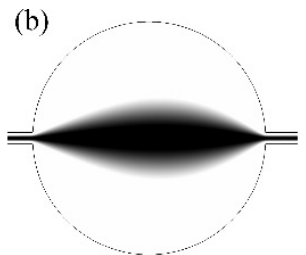

(i)

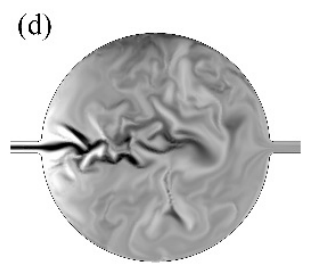

(i)

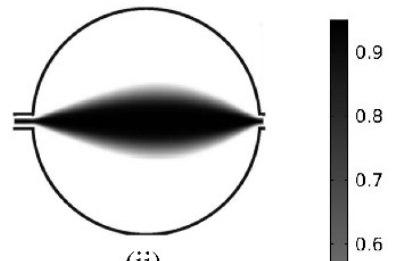

(ii)

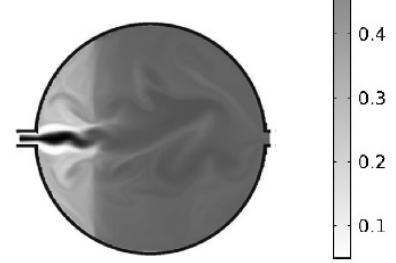

(ii)

313 FIG. 4 Concentration contour of (a) ferrofluid-cladding configuration without magnetic field (b)

314 ferrofluid-core configuration without magnetic field, (c) ferrofluid-cladding configuration with

315 the magnetic field and (d) ferrofluid-core configuration with the magnetic field. (i) present

316 results (ii) Zhu and Nguyen study ${ }^{35}$. Reproduced from G.-P. Zhu, and N.-T. Nguyen, "Rapid

317 magnetofluidic mixing in a uniform magnetic field," Lab on a Chip 12, 4772 (2012), with the

318 permission of Royal Society of Chemistry Publishing.

\section{B. Mixing enhancement in droplet-based micro magnetofluidics}

\section{Materials and Methods}

322 Fig. 5 illustrates the schematic of our proposed model. The main simulation domains include a 323 disperse-phase flow containing two miscible fluids, a continuous-phase flow, and surrounding 324 media. Droplets are generated and merged in a double T-junction device with a straight main 325 channel and two horizontally symmetric side channels. Considering the shallow microchannels 326 (with 50 depths and the aspect ratio of 3), two-dimensional (2D) model is employed for numerical 327 simulation. The two miscible disperse-phase fluids flow through the side channels (water-based 328 ferrofluid from the lower channel and a diamagnetic glycerol-water mixture from the upper 329 channel) while the continuous fluid (oil), flows through the main channel. Considering water330 based ferrofluid, the solubility of magnetic nanoparticles inside the oil is near zero, which results 
331 in a concentration jump across the ferrofluid-oil interface. Besides, merging of two miscible phases

332 establishes additional concentration gradient inside the droplet. After applying a uniform magnetic

333 field, the existing concentration gradients creates an extra magnetic force. To provide

334 experimentally replicable results, the materials properties were extracted from the similar

335 experimental studies. The magnetization saturation of the ferrofluid is $32.5 \mathrm{mT}$, its density is

$336 \rho_{\mathrm{ff}}=1.1 \times 10^{3} \mathrm{~kg} \mathrm{~m}^{-3}$ and its viscosity is $v_{\mathrm{ff}}=5 \mathrm{mPa} \cdot \mathrm{s}$. The initial volumetric concentration and

337 the initial susceptibility of the ferrofluid are set as $2 \%$ and $\chi_{\mathrm{ff}}=0.36$, respectively. The

338 diamagnetic mixture is considered to be made of $65.6 \mathrm{wt} \%$ deionized water and $34.4 \mathrm{wt} \%$ glycerol.

339 Consequently, the mixture has the viscosity and the density of $v_{\mathrm{m}}=2.5 \mathrm{mPa} \cdot \mathrm{s}$ and

$340 \rho_{\mathrm{m}}=1.09 \times 10^{3} \mathrm{~kg} \mathrm{~m}^{-3}$, respectively. ${ }^{35}$ The viscosity and density of the oil are $\rho_{o}=960 \mathrm{~kg} \mathrm{~m}^{-3}$ and

$341 v_{o}=96 \mathrm{mPa} \cdot \mathrm{s}$. The Marangoni effect is ignored and a constant value of $\sigma=12 \mathrm{mN} / \mathrm{m}$ is used

342 for both oil-ferrofluid and oil-mixture interfacial tensions. ${ }^{40,49,50}$ Given that the two miscible fluids

343 have very close properties, it would be a valid assumption to ignore the Marangoni effect in the

344 solution as frequently being assumed in previous works on mixing inside droplets. ${ }^{28,}{ }^{42}$ The

345 diffusion coefficients of magnetic nanoparticles into the water-glycerol mixture, as well as, oil are

346 obtained from Einstein's model as $D=k_{b} T / 3 \pi \eta d_{p}$, where $D$ shows diffusion coefficient, $k_{b}$

347 Boltzmann constant, $T$ absolute temperature and $d_{m p}$ the magnetic particles diameter. Considering

348 the temperature of $300 \mathrm{~K}$ and $10 \times 10^{-9} \mathrm{~m}$ particles diameter, the diffusion coefficients of magnetic

349 particles into the mixture and the oil are calculated as $1.76 \times 10^{-11}$ and $4.58 \times 10^{-13}$, respectively.

350 To describe the dynamic of a two-phase flow, as well as the influence of the magnetic forces on 
351 the inhomogeneous ferrofluid, we utilized the coupled systems of Maxwell equations, advection-

352 diffusion equation, incompressible Navier-Stokes equation and the Young-Laplace equation. ${ }^{39}$

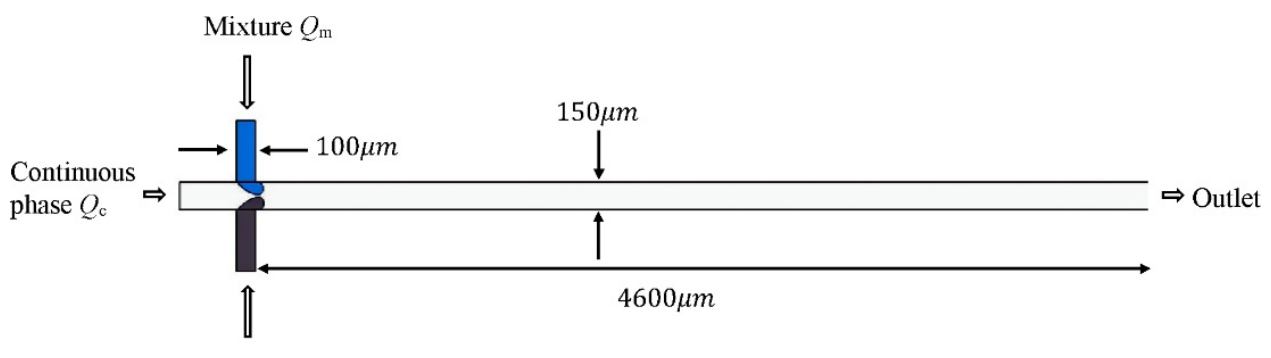

Ferrofluid $Q_{\text {ff }}$

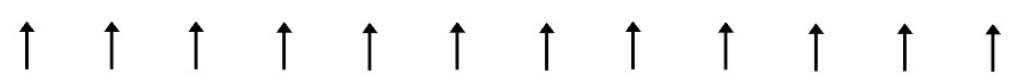

354 FIG. 5 Schematic of the droplet-based micromagnetofluidic for mixing enhancement inside the 355 microdroplets.

356 The effective dimensionless numbers in this study are Reynolds, Peclet and Capillary numbers.

357 Reynolds number is the ratio of the inertia force to the viscous force. This number determines the

358 flow regime inside the channel. Because of the tiny microchannels, the Reynolds number all over

359 this study is very smaller than one, so the flow regime is laminar. Peclet number shows the ratio

360 of convective mass transport to diffusive mass transport. For high Peclet numbers, a substantial

361 proportion of the mass transport is carried out by convection. Also, Capillary number plays a

362 pivotal role in droplet generation regime. This number shows the ratio of the viscous forces to the

363 interfacial tension. For double T-junction devices, previous studies have classified droplet

364 formation regimes according to the Capillary number, as well as, dispersed phase volume fraction

365 to four subcategories: merging, alternating, small droplets, and laminar regimes. According to

366 these studies, for a constant value of the dispersed phase volume fraction, as the Capillary number

367 increases the droplet formation regime changes from merging to laminar. ${ }^{51,52}$ 
368 In the present study, we also explore the influence of key parameters such as magnetic field 369 strength, droplets mean velocity and droplets size on mixing enhancement, systematically. To 370 verify the effect of the magnetic field on mixing behavior, a magnetic field varying from $10 \mathrm{mT}$ 371 to $90 \mathrm{mT}$ is exploited in the simulation. For this case, the flow rate of the oil is set as $0.09 \mathrm{ml} / \mathrm{h}$, 372 and the flow rates of each of dispersed fluids (ferrofluid and the mixture) is $0.009 \mathrm{ml} / \mathrm{h}$. The 373 corresponding Reynolds number $\left(\operatorname{Re}=\rho_{c} u_{c} L / \eta_{c}, L\right.$ is the width of the main channel), Capillary 374 number $\left(C a=\eta_{c} u_{c} / \sigma\right)$ and Peclet number $\left(\mathrm{Pe}=u_{s} l_{s} / D_{m p}, u_{s}\right.$ is the velocity of the slug, $l_{s}$ is the 375 length of the slug and $D_{m p}$ is diffusion coefficient of the magnetic particle to the diamagnetic 376 mixture) are $\mathrm{Re}=5 \times 10^{-3}, \mathrm{Ca}=2.67 \times 10^{-2}, \mathrm{Pe}=6.57 \times 10^{4}$.

377 The other verified parameter is the mean velocity of droplets in the main channel. We kept the 378 aspect ratio of the flow rates fixed $\left(Q_{r}=Q_{c} / Q_{d}=3.33, Q_{d}=Q_{\mathrm{ff}}+Q_{\mathrm{m}}\right)$ and altered their 379 magnitudes, so that the flow rates of the continuous and dispersed phases vary from 0.096 to 0.144 $380 \mathrm{ml} / \mathrm{h}$ and 0.0288 to $0.0432 \mathrm{ml} / \mathrm{h}$, respectively $\left(1.0 \leq Q_{v}=Q / Q_{0} \leq 1.5, Q_{v}\right.$ is the dimensionless 381 value of flow rate that is obtained by dividing the flow rate to the initial flow rate). The 382 corresponding ranges of the Reynolds number are from $5.33 \times 10^{-3}$ to $8.0 \times 10^{-3}$, the ranges of the 383 Capillary number are from $2.84 \times 10^{-2}$ to $4.27 \times 10^{-2}$ and the ranges of the Peclet number are from $3845.93 \times 10^{4}$ to $8.43 \times 10^{4}$.

385 Finally, the effect of the droplet size on mixing is investigated by shifting the dispersed phase 386 flowrate from $0.018 \mathrm{ml} / \mathrm{h}$ to $0.09 \mathrm{ml} / \mathrm{h}$ and maintaining the continuous phase flowrate constant at 387 $0.12 \mathrm{ml} / \mathrm{h}$. Accordingly, the corresponding Reynolds number and Capillary number are $6.67 \times 10^{-3}$ 388 and $3.56 \times 10^{-2}$, respectively. Also, the ranges of the Peclet number is from $5.12 \times 10^{4}$ to $1.3 \times 10^{5}$ 
389 The above-mentioned circumstances, suggest laminar flow because of the low Reynold numbers, 390 small diffusion because of the high Peclet numbers and merging regime of droplet formation due 391 to the low Capillary number.

\section{2. Mesh convergence}

393 In all the geometries, three-dimensional tetrahedral grids were used for meshing. Mesh 394 independency was examined by three case studies $(19,266 ; 33,052$ and 47,264 number of grids). 395 In the channel domain, the mesh was highly smoothed in all cases with a maximum element growth 396 rate of 1.08. Fig. 6 (a) and (b) show the yielded droplet shapes and particles concentration 397 distribution for all these cases. Negligible changes were observed when grids number went beyond 39833,052 . In the present study, 47,264 elements were used for meshing. 


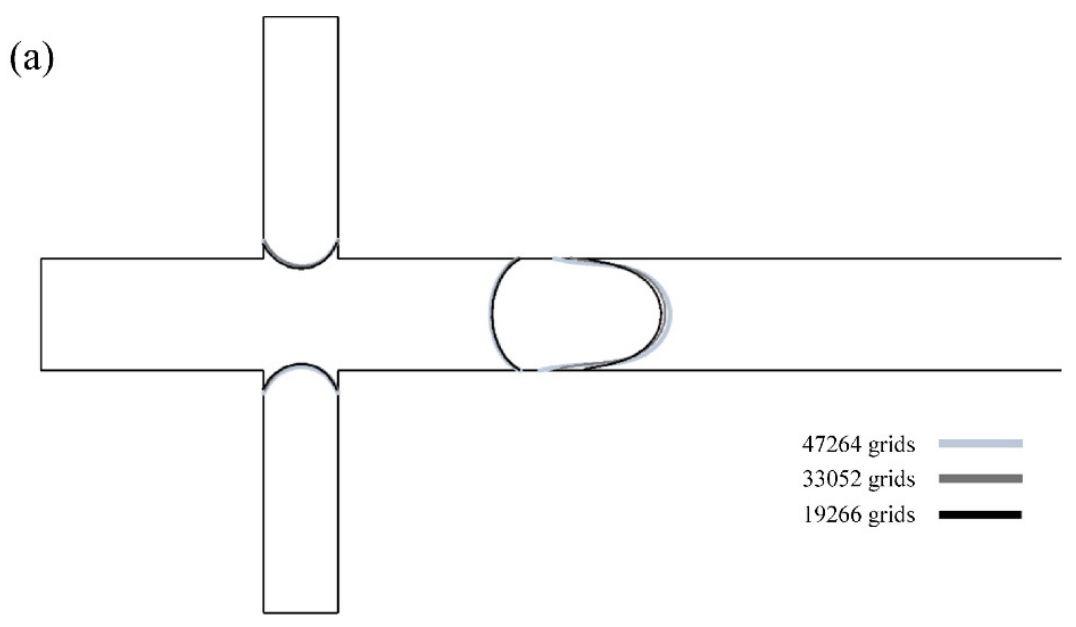

(b)

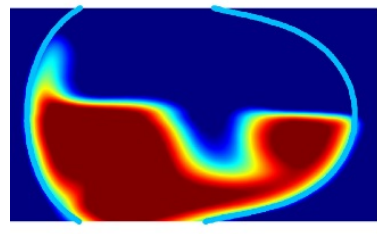

(i)

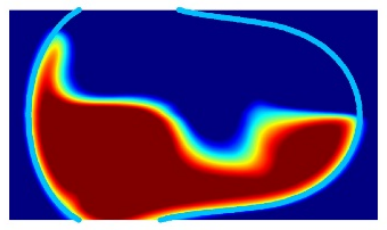

(ii)

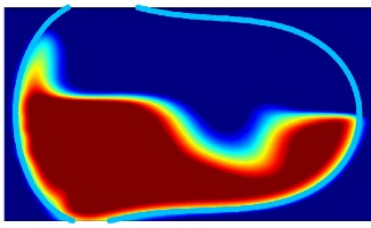

(iii)

400 FIG. 6 Mesh convergence study (a) droplet shapes for a different number of grids (b) particles 401 concentration distribution for (i) 19266 grids (ii) 33052 grids (iii) 47264 grids $\left(Q_{\mathrm{c}}=0.09 \mathrm{ml} / \mathrm{h}\right.$,

$\left.402 Q_{\mathrm{ff}}=Q_{\mathrm{m}}=0.009 \mathrm{ml} / \mathrm{h}, \mathbf{B}=0 \mathrm{mT}\right)$.

403

404

\section{Droplet formation in the double T-junction device}

405 As discussed previously, a double T-junction configuration is considered for droplet forming and

406 merging. ${ }^{51,52}$ This configuration operates in four different regimes, which are characterized by the

407 capillary number and dispersed phase volume fraction. The present study implements small

408 capillary number and small volume fraction of the dispersed phase. Thus, merging of dispersed

409 phases occurs at the very beginning of the main channel. However, other scenarios for droplet

410 merging and mixing are possible. For instance, droplet merging and mixing can be realized in an

411 alternative regime by employing a suitable magnetic field configuration. 
412 The mechanism of the droplet formation is such that in the early growth stage of the dispersed

413 phases, the bulged part of the droplet grows both horizontally and vertically. Meanwhile, the gap

414 between two dispersed phases reduces, leading to a narrower film of the immiscible phase. Finally,

415 the film ruptures and the two miscible phases coalescence. According to the previous studies, the

416 small values of the viscosities of the dispersed phases improve surface mobility and make merging

417 easier. Also, large values of the interfacial tensions speed up coalescing. ${ }^{51}$ After coalescence, the

418 main channel is blocked with dispersed phases. The blockage increases the pressure upstream of

419 the cross junction. The growth of the disperse phase in the channel continuously enlarges the

420 pressure gradient across the droplet. Finally, the pressure gradient overcomes the pressure inside

421 the dispersed phase and squeezes the interface. The generated droplet is confined by channel walls

422 and adapts a plug shape ${ }^{53,54}$. Any increase of the flow rate of the continuous phase amplifies the

423 pressure gradient in the channel and accelerates the droplet formation. After a threshold value,

424 increasing of the continuous phase flow rate makes the dispersed phases unable to rupture the film

425 and droplet generation regime changes.

426 Our results show a slight delay in ferrofluid detachment compared to the diamagnetic mixture 427 detachment (Fig. 7), which is consistent with previous researches offering that an increase in 428 dispersed phase viscosity, elongates the neck and postpones the detachment. ${ }^{51}$ This delay in 429 detachment contributes to the fluids distribution inside the droplet, right after the formation. 430 Particles concentration contours during droplet formation process without magnetic field and 431 under a uniform magnetic field of $90 \mathrm{mT}$ strength are demonstrated in Figs. 7(a) and 7(b) 432 respectively. It is noteworthy that the detachment of the mixture fluid is at the same time for both 433 cases, while the ferrofluid with magnetic field detaches in a shorter time compared to without 434 magnetic field. This issue can be attributed to the internal hydrodynamic of the droplet. Under the 
435 influence of the magnetic field, the ferrofluid rises at the center rear part of the droplet, which 436 results in drainage of the ferrofluid neck and acceleration of the ferrofluid detachment.

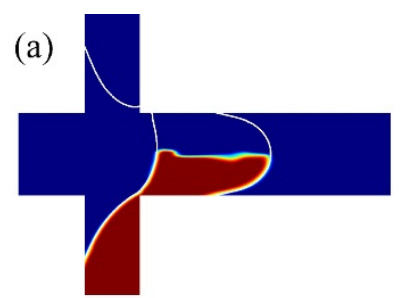

$\mathrm{t}=102 \mathrm{~ms}$

(b)

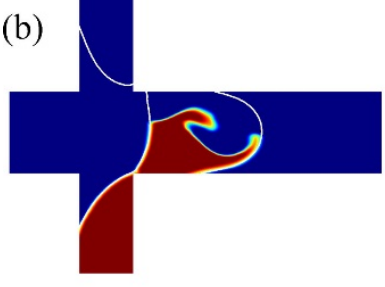

$\mathrm{t}=102 \mathrm{~ms}$

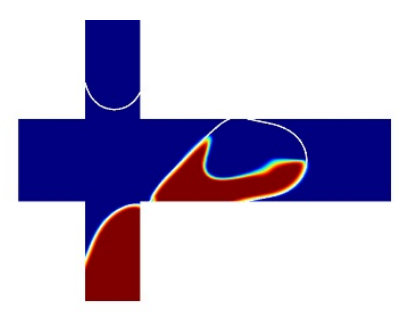

$\mathrm{t}=116 \mathrm{~ms}$

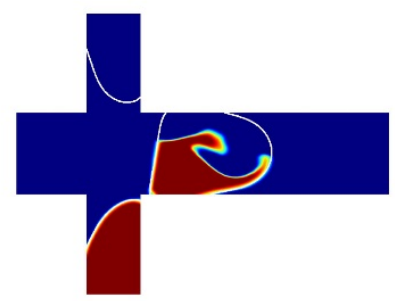

$\mathrm{t}=103 \mathrm{~ms}$

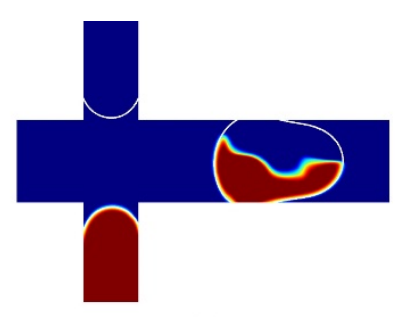

$\mathrm{t}=130 \mathrm{~ms}$

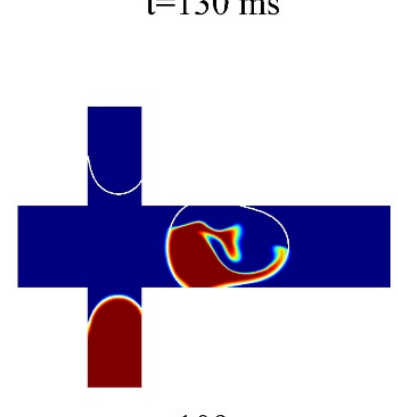

$\mathrm{t}=109 \mathrm{~ms}$

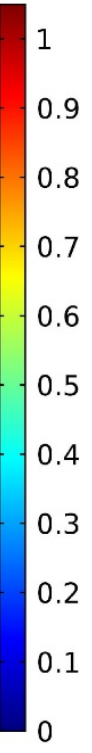

439 FIG. 7 concentration contour during droplet formation process (a) without magnetic field (b) 440 subjected to a magnetic field with $90 \mathrm{mT}$ strength.

441 4. Mixing efficiency inside droplets under different magnetic field strengths

442 Figs. 8 and 9 illustrates th mixing behavior inside moving droplets for different values of magnetic

443 field strengths. According to these results, in the early stages of increasing the magnetic field, the 444 mixing efficiency inside droplets increases rapidly. The efficiency growth then diminishes 445 gradually, and after a specific value, any increase of the magnetic field strength has an adverse 446 effect on mixing efficiency. In the absence of the magnetic field, the flow topology inside the 447 droplet is influenced by parameters such as fluids properties, channel geometry, droplet size, and 448 droplet velocity. ${ }^{55,56}$ For plug shape droplets, a thin film of the continuous phase is formed between 449 the droplet and the channel wall which exerts shear forces on droplet sides. Under these 450 circumstances, the difference between droplet velocity and mean velocity in the channel results in 
451 the emergence of horizontally symmetrical internal recirculation in the droplet. ${ }^{57}$ Considering the 452 lower half of the droplet, during droplet movement inside the channel a clockwise vortex develops 453 in the droplet flanks that accompanied two weaker counter-clockwise vortices in the rear and front 454 parts (Fig. $10(a-i)$ and (b-i)). This pattern of the internal flows is maintained throughout the droplet 455 traveling in a straight channel.

456 Droplets exposed to the uniform magnetic field experience magnetic force in two ways. First, 457 because of the distinct solubility of magnetic particles in two immiscible phases and their 458 concentration difference, the magnetic force acts at their interface and elongates the droplet in line 459 with the magnetic field. Moreover, once the miscible phases merge, a sharp concentration gradient 460 exists at their interface. The established magnetic force due to the susceptibility gradient within 461 the droplet has the most impact on the internal flow patterns. After applying the magnetic field, 462 depending on the strength of the field, the existing magnetic susceptibility gradient at the interface 463 of miscible phases gives rise to two different mixing behaviors, Figs. 6(b-e).

464 At low magnetic fields, the magnetophoresis improves nanoparticles migration from the high 465 concentration to the low concentration regions. Under this condition, the dominant mode of 466 particles transport is diffusion. On the other hand, If the magnetic field becomes large enough, the 467 magnetic force disturbs the symmetry of the internal droplet flows and produces secondary flows. 468 That way, as long as the susceptibility gradient parallel to the magnetic field is sufficiently large, 469 the number and size of vortices inside the droplets are constantly changing. Here the particles 470 transportation is mainly realized through the bulk flow convection, which leads to a considerable 471 enhancement of mixing. ${ }^{35}$ After droplet formation, most of the particles are trapped within the 472 lower half recirculation zones of the droplet, which are hydrodynamically isolated from the upper 473 ones. Weak magnetic fields only improve diffusive behaviors of the particles, so they have a small 
474 influence on mixing improvement, Fig. 8(b) and 9. With augmenting the magnetic field strength,

475 it is possible to create secondary flows in the regions with sharp concentration gradients; however,

476 once the gradient is reduced, the magnetic force is attenuated and unable to perturbs the symmetry

477 of the recirculation flows any longer, Fig. 8(c).

478 Increasing the magnetic field strength enhances the particles convection in the droplet. Because of 479 the nearly complete predominance of the magnetic force to other driving forces, particles are 480 transported in the magnetic field direction swiftly, and distributed at the rear center part of the 481 droplet vertically, Fig. 8 (d) and (e). Intensifying of the magnetic field accelerates particles 482 convection and increases the volume of the transported particles. After homogenization of particles 483 in the same direction of the magnetic field and reduction of the magnetic force, the vortices inside 484 the droplet turn to its symmetrical configuration (Fig. 10 (a-ii), t=264 ms). In this instance, the 485 droplet middle vortices are smaller than that of the zero magnetic field case. However, these 486 vortices gradually grow as the droplet moves within the channel and particles diffuse toward the 487 droplet leading edge. During this process, exerting shear forces proceeded by droplet movement 488 tend to enlarge the vortices in the droplet flanks, whereas particles under the influence of the 489 uniform magnetic field resist deviation from the homogenized state.

490 As the stronger magnetic force is employed, the effect of this resistance is more pronounced. As 491 such, the strong magnetic force slows down the variation of the middle part vortices and reduces 492 the homogenization, Fig. 8(e) and Fig. (9). Fig. (9) clearly shows that mixing performance in 493 droplets exposed to magnetic field consists of two steps. At the beginning of the droplet movement 494 in the channel, the variation of the efficiency with the time has a steep slope that increases with 495 increasing the magnitude of the magnetic field. This behavior is due to the massive particle 496 convection by magnetic forces when a sharp susceptibility gradient exists in the droplet. After 
497 dropping of the gradient in magnetic field direction, the main driving force of the mass transport 498 is diffusion which acts much slower than convective mass transport, so the slope declines 499 significantly.

500 Figure 10 shows the streamlines of internal flows in the droplet frame of reference. The figure is 501 related to the initial steps of the droplet movement within the channel for two cases of without 502 magnetic field and in a 90-mT magnetic field. As observed in Fig. 10 (a-i), for the case of zero 503 magnetic field, at the very beginning after the droplet formation, the recirculation patterns and 504 particles distribution inside the droplet is asymmetrical. The main reason for the imbalance is the 505 difference in the fluids properties, which affect the droplet formation process. However, as 506 discussed above, shortly after the droplet movement in the channel, the symmetrical pattern of the 507 recirculation flows appears inside the droplet. At the initial steps of applying the magnetic field, a 508 large vortex developed in the center of the droplet due to the intense magnetic force, Fig. 10 (a-ii) 509 ( $t=115 \mathrm{~ms})$. The vortex transports a remarkable volume of particles vertically and moves them 510 toward the rear part of the droplet, Fig. 10 (a-ii) ( $\mathrm{t}=115-154 \mathrm{~ms})$. Meanwhile, new vortices emerge 511 in the middle of the droplet. Then, the large vortex divides into new vortices, and the internal flows 512 advance toward their symmetrical shapes. After the formation of the symmetrical vortices, the 513 majority of the particles are isolated at the middle and rear vortices. These particles transport to 514 the droplet head through the diffusion, as well as, expansion of the middle vortices. 
(a)

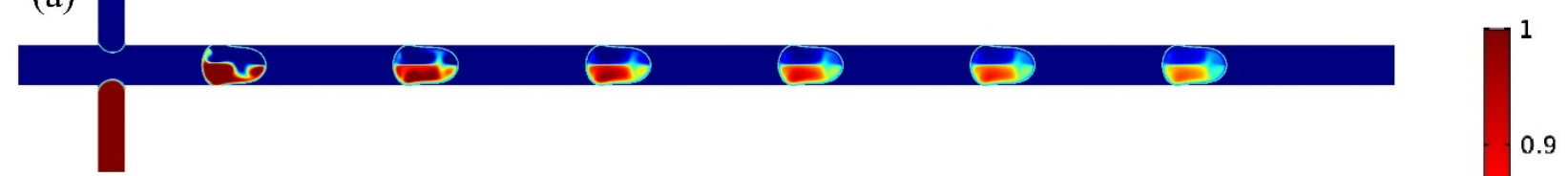

(b)

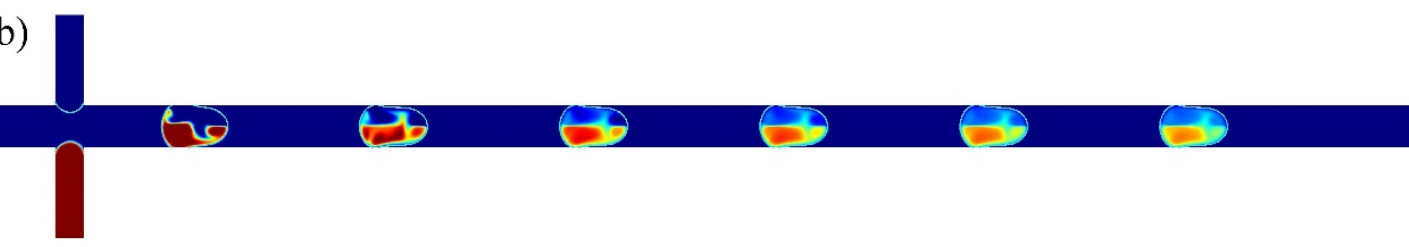

(c)

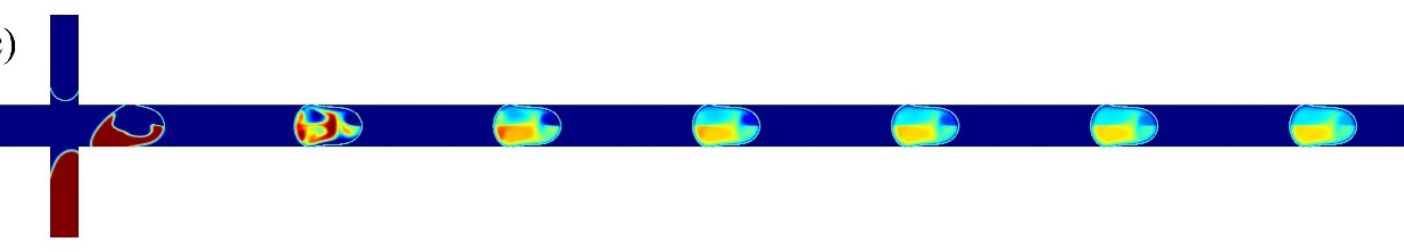

(d)

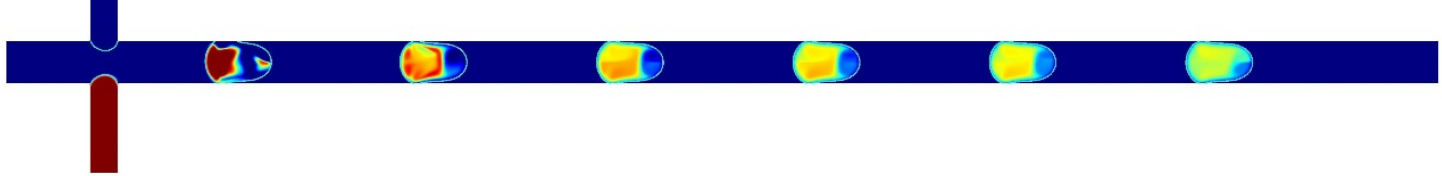

(e)

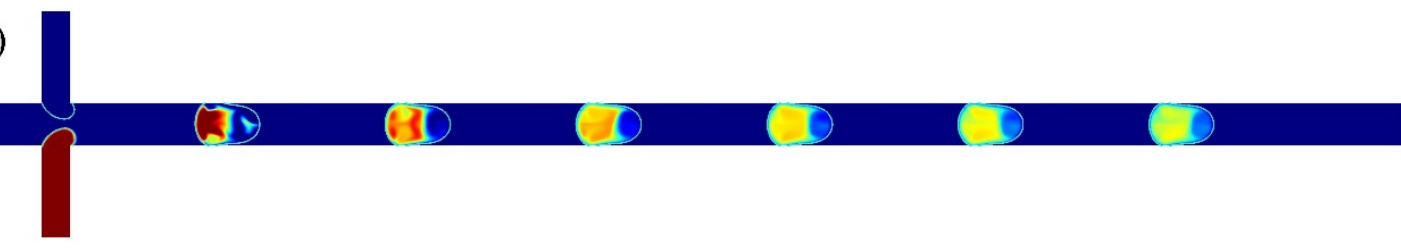

518 FIG. 8 Nanoparticles concentration distribution in micro droplets under influence of the magnetic 519 fields with (a) $0 \mathrm{mT}$ (b) $10 \mathrm{mT}$ (c) $30 \mathrm{mT}$ (d) $60 \mathrm{mT}$ and (e) $90 \mathrm{mT}$ strengths ( $\left.520 Q_{\mathrm{ff}}=Q_{\mathrm{m}}=0.009 \mathrm{ml} / \mathrm{h}, Q_{\mathrm{c}}=0.09 \mathrm{ml} / \mathrm{h}\right)$. 


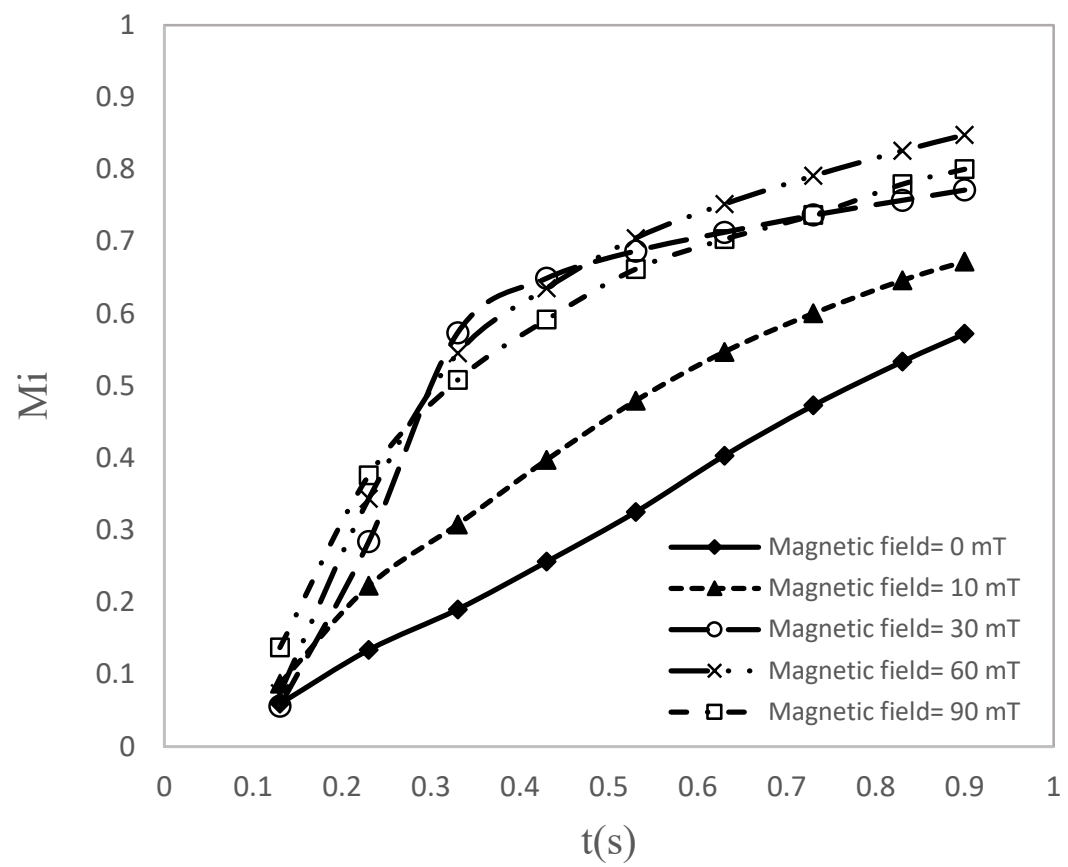

522

523 FIG. 9 Mixing efficiency in droplet versus time for different values of the magnetic field strengths $524\left(Q_{\mathrm{ff}}=Q_{\mathrm{m}}=0.009 \mathrm{ml} / \mathrm{h}, Q_{\mathrm{c}}=0.09 \mathrm{ml} / \mathrm{h}\right)$.

525 
(i)

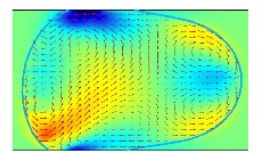

$\mathrm{t}=127 \mathrm{~ms}$

(ii)

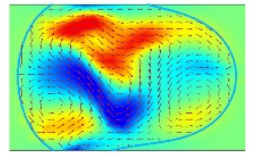

$\mathrm{t}=115 \mathrm{~ms}$

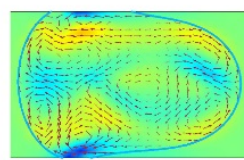

$\mathrm{t}=161 \mathrm{~ms}$

(i)

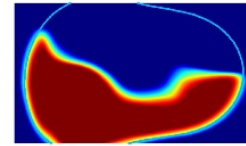

$\mathrm{t}=127 \mathrm{~ms}$

(ii)

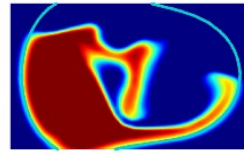

$\mathrm{t}=115 \mathrm{~ms}$

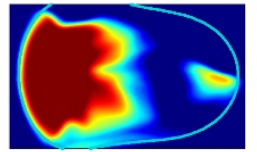

$\mathrm{t}=161 \mathrm{~ms}$

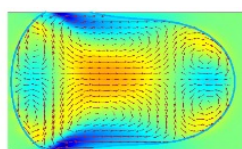

$\mathrm{t}=150 \mathrm{~ms}$

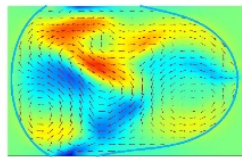

$\mathrm{t}=127 \mathrm{~ms}$

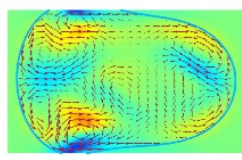

$\mathrm{t}=179 \mathrm{~ms}$

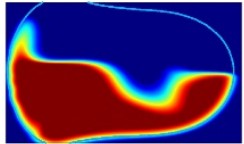

$\mathrm{t}=150 \mathrm{~ms}$

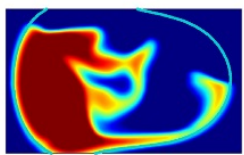

$\mathrm{t}=127 \mathrm{~ms}$

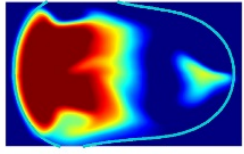

$\mathrm{t}=179 \mathrm{~ms}$

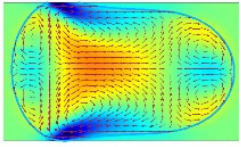

$\mathrm{t}=179 \mathrm{~ms}$

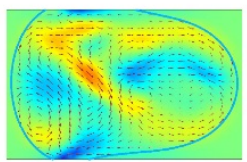

$\mathrm{t}=138 \mathrm{~ms}$

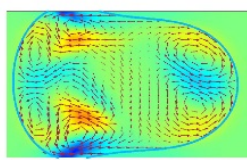

$\mathrm{t}=220 \mathrm{~ms}$

(a)

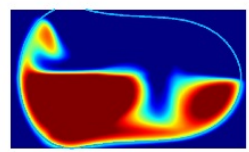

$\mathrm{t}=179 \mathrm{~ms}$

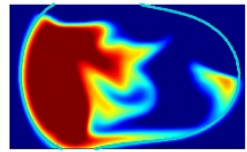

$\mathrm{t}=138 \mathrm{~ms}$

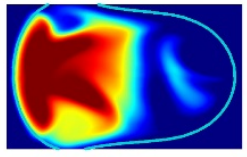

$\mathrm{t}=220 \mathrm{~ms}$

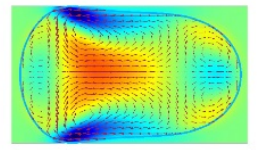

$\mathrm{t}=264 \mathrm{~ms}$

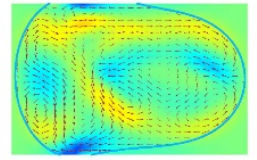

$\mathrm{t}=150 \mathrm{~ms}$

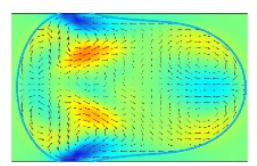

$\mathrm{t}=264 \mathrm{~ms}$

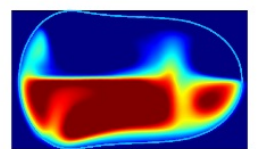

$\mathrm{t}=264 \mathrm{~ms}$

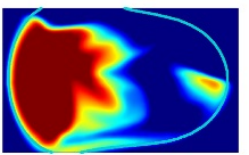

$\mathrm{t}=150 \mathrm{~ms}$

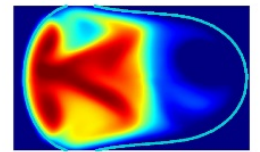

$\mathrm{t}=264 \mathrm{~ms}$

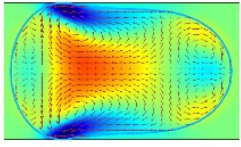

$\mathrm{t}=325 \mathrm{~ms}$

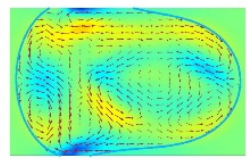

$\mathrm{t}=154 \mathrm{~ms}$

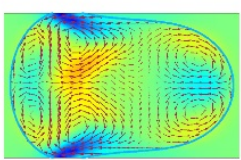

$\mathrm{t}=325 \mathrm{~ms}$

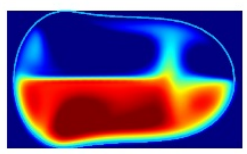

$\mathrm{t}=325 \mathrm{~ms}$

$\mathrm{t}=154 \mathrm{~ms}$

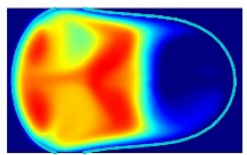

$\mathrm{t}=325 \mathrm{~ms}$
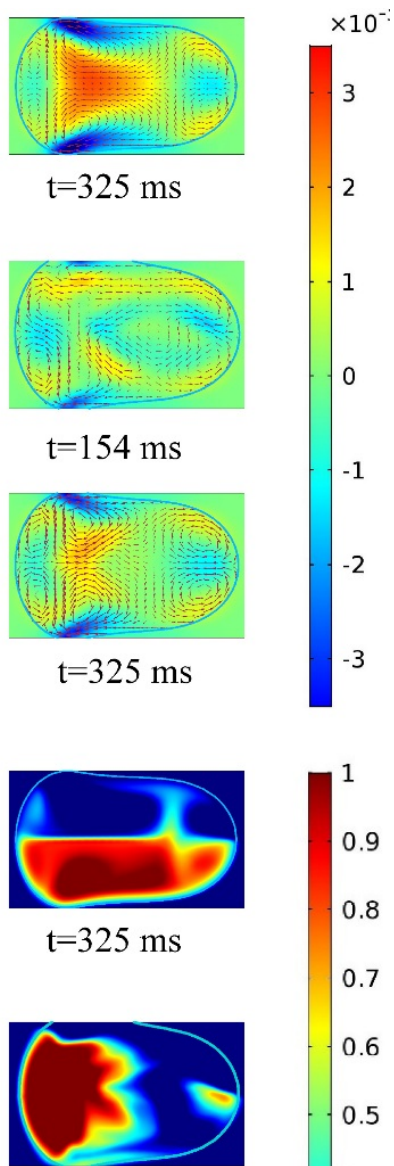

(b)
526
527

528

529

530

531

532

533

534

\section{Mixing efficiency inside droplets with different mean velocities}

FIG. 10 Mixing performance of droplet at initial steps of droplet moving in microchannel (a) relative velocities and streamlines in the droplet frame of the reference, (b) nanoparticles concentration distribution inside the droplets for (i) without magnetic field, and (ii) exposed to a magnetic field with $90 \mathrm{mT}$ strength $\left(Q_{\mathrm{ff}}=Q_{\mathrm{m}}=0.009 \mathrm{ml} / \mathrm{h}, Q_{\mathrm{c}}=0.09 \mathrm{ml} / \mathrm{h}\right)$.

To evaluate the influence of the droplet velocities on the mixing efficiency, we kept the aspect ratio of the flowrates fixed $\left(Q_{r}=Q_{c} / Q_{d}=3.33\right)$ and varied their magnitudes ( 
$5351.0 \leq Q_{v}=Q / Q_{0} \leq 1.5$ ). Figs. 11 and 12 illustrate the mixing process and efficiency within the 536 microdroplets for different values of $Q_{v}$. At low mean velocities, the magnetic force rapidly 537 distorts the vortices and accumulates a large volume of the particles at the rear center of the droplet, 538 Fig. 11(a). Increasing the droplets velocity intensifies viscous force and accordingly strengthens 539 vortices inside the droplets. As a result, the boosted vortices resist to change under the same 540 magnetic force (peculiarly middle vortices, which have the most strength). Subsequently, the 541 magnetic force develops the recirculation zones containing nanoparticles towards the weaker 542 vortex at the upper front part of the droplet. The magnetic force pulls the particles upward and 543 shrinks the upper middle vortex. Because of the relatively high strength, the shrinkage of the upper 544 middle vortex is insignificant, and a part of the particles move toward the droplet tip. At the 545 intermediate conditions, balancing between magnetic forces and shear forces caused by droplet 546 movement in the channel leads to an approximately uniform particles distribution all over the 547 droplet and enhances mixing, remarkably, so that mixing efficiency can reach more than $94 \%$, 548 Figs. 11(b) and 12. Further increasing the droplet velocity prevents the deformation of the upper 549 middle vortex and makes particles convection to this region difficult. Thus, most of the particles 550 remain confined in the lower vortices or move to the droplet tip. This status causes a heterogeneity 551 in particles distribution, which exacerbates with increasing droplet velocity, Figs. 11(c-e) and 12. 


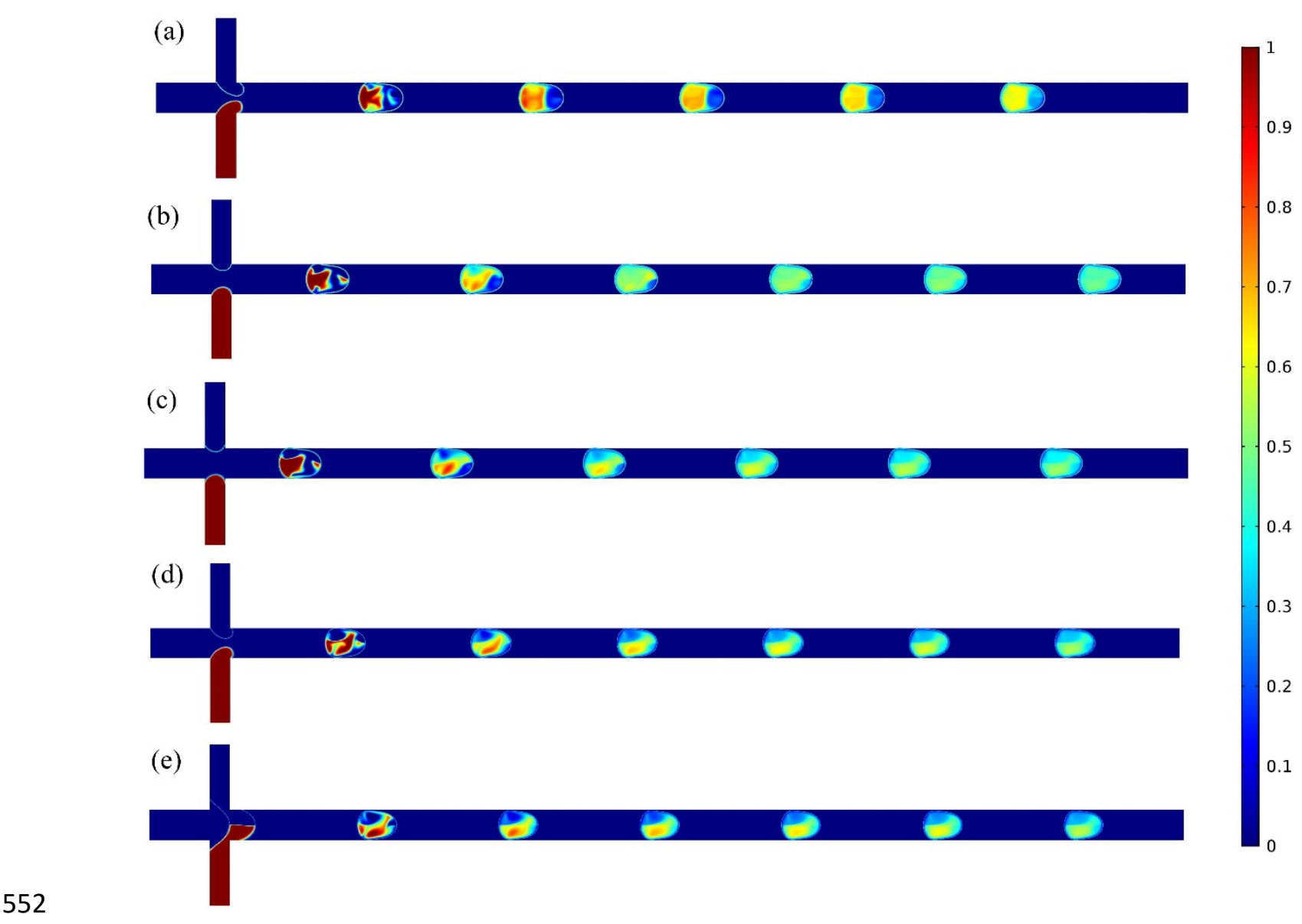

553 FIG. 11 Nanoparticles concentration distribution in microdroplets with various average velocities 554 (a) $Q_{v}=1$, (b) $Q_{v}=1.125$, (c) $Q_{v}=1.25$, (d) $Q_{v}=1.375$ and (e) $Q_{v}=1.5 \quad(\mathbf{B}=60 \mathrm{mT}$, $\left.555 \quad 0.096 \leq Q_{c} \leq 0.144 \mathrm{ml} / \mathrm{h}, 0.096 \leq Q_{c} \leq 0.144 \mathrm{ml} / \mathrm{h}\right)$.

556 


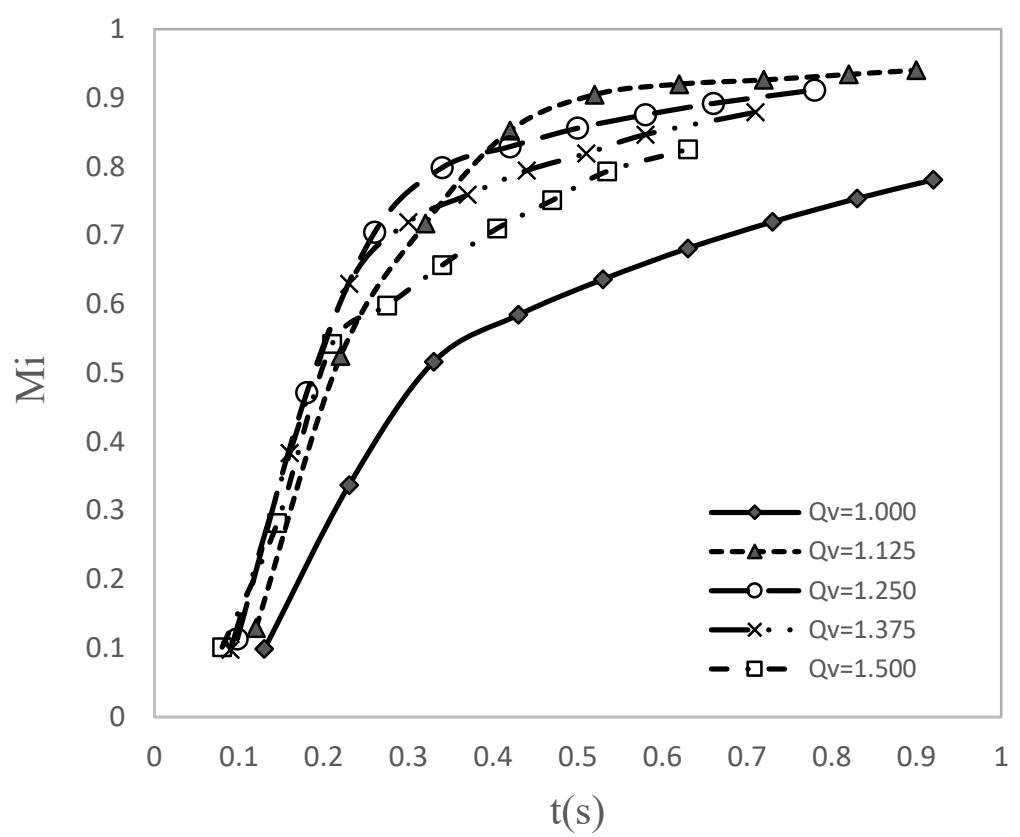

559 FIG. 12 Mixing efficiency in droplet versus time for different values of the average droplet 560 velocities $\left(\mathbf{B}=60 \mathrm{mT}, 0.096 \leq Q_{c} \leq 0.144 \mathrm{ml} / \mathrm{h}, 0.096 \leq Q_{c} \leq 0.144 \mathrm{ml} / \mathrm{h}\right)$.

561 Figure 13(a) demonstrates the streamlines and velocity contours inside droplets with different 562 mean velocities, where the center-to-center distance between the droplet and the cross junction is 563 nearly 250 micrometers. Also, the related concentration contours are represented in Fig. 13(b), 564 which clearly shows that increasing droplet velocity hampers upper middle vortex deformation, 565 and causes convection of particles towards the droplet tip. 


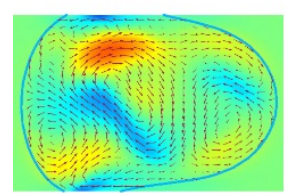

$Q_{m}=1.000$

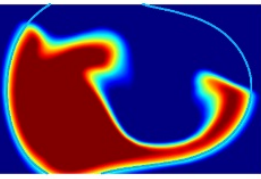

$Q_{m}=1.000$

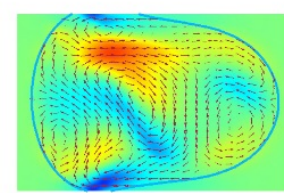

$Q_{m}=1.125$

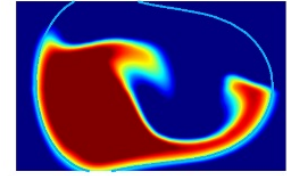

$Q_{m}=1.125$

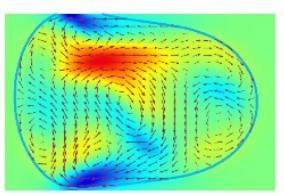

$Q_{m}=1.250$

(a)

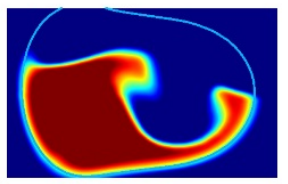

$Q_{m}=1.250$

(b)

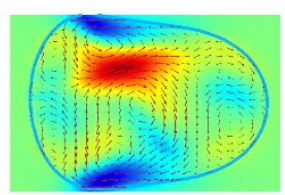

$Q_{m}=1.375$

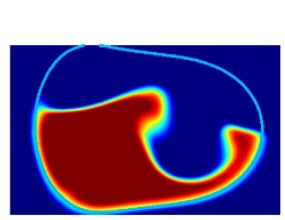

$Q_{m}=1.375$

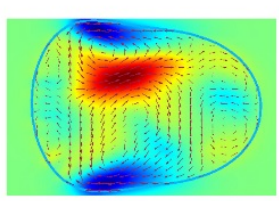

$Q_{m}=1.500$
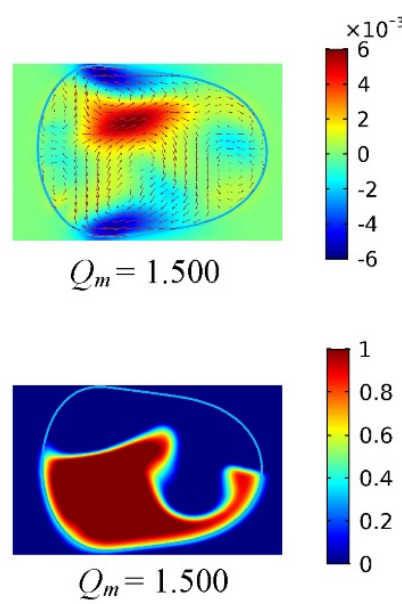

FIG. 13 Mixing performance of droplets with various droplet average velocities and 250 micrometers center to center distance from the cross junction, (a) relative velocities and streamlines in the droplet frame of the reference, (b) nanoparticles concentration distribution inside the droplets $\left(\mathbf{B}=60 \mathrm{mT}, 0.096 \leq Q_{c} \leq 0.144 \mathrm{ml} / \mathrm{h}, 0.096 \leq Q_{c} \leq 0.144 \mathrm{ml} / \mathrm{h}\right)$.

\section{Mixing efficiency inside droplets with different volumes}

Another influencing factor on mixing efficiency is the droplet size. To verify the influence of droplet volume on mixing efficiency, we used different values of dispersed phase flow rates. For large flow rates of the dispersed phase, volume and speed of the droplets increases, and their distance gets smaller. Under these circumstances, the middle part vortices of droplets become large and strong, while other vortices (rear and front part vortices) change slightly (Fig. 16). According to the previous arguments, as the vortices strengths increase, more energy is required to deform them. Hence, as the droplets are getting bigger, the magnetic field has a weaker influence on the flank vortices. As a result, the magnetic field steers particles toward weaker vortices and their boundaries. The decrease of the concentration gradient inside the droplets progressively brings the vortices shapes closer to their symmetrical states. Meanwhile, the upper middle vortex is developing downward and takes a part of the particles from this region. As initial changes of vortices become larger, more particles get stuck in upper middle vortex following their 
584 development. These particles follow the rotational flow of the vortex, which improves mixing 585 inside the droplets. In small droplets, mass transport between these vortices takes place more 586 conveniently because of the narrower boundary between droplet middle and tip vortices, Figs. 14 587 and 16 .

588

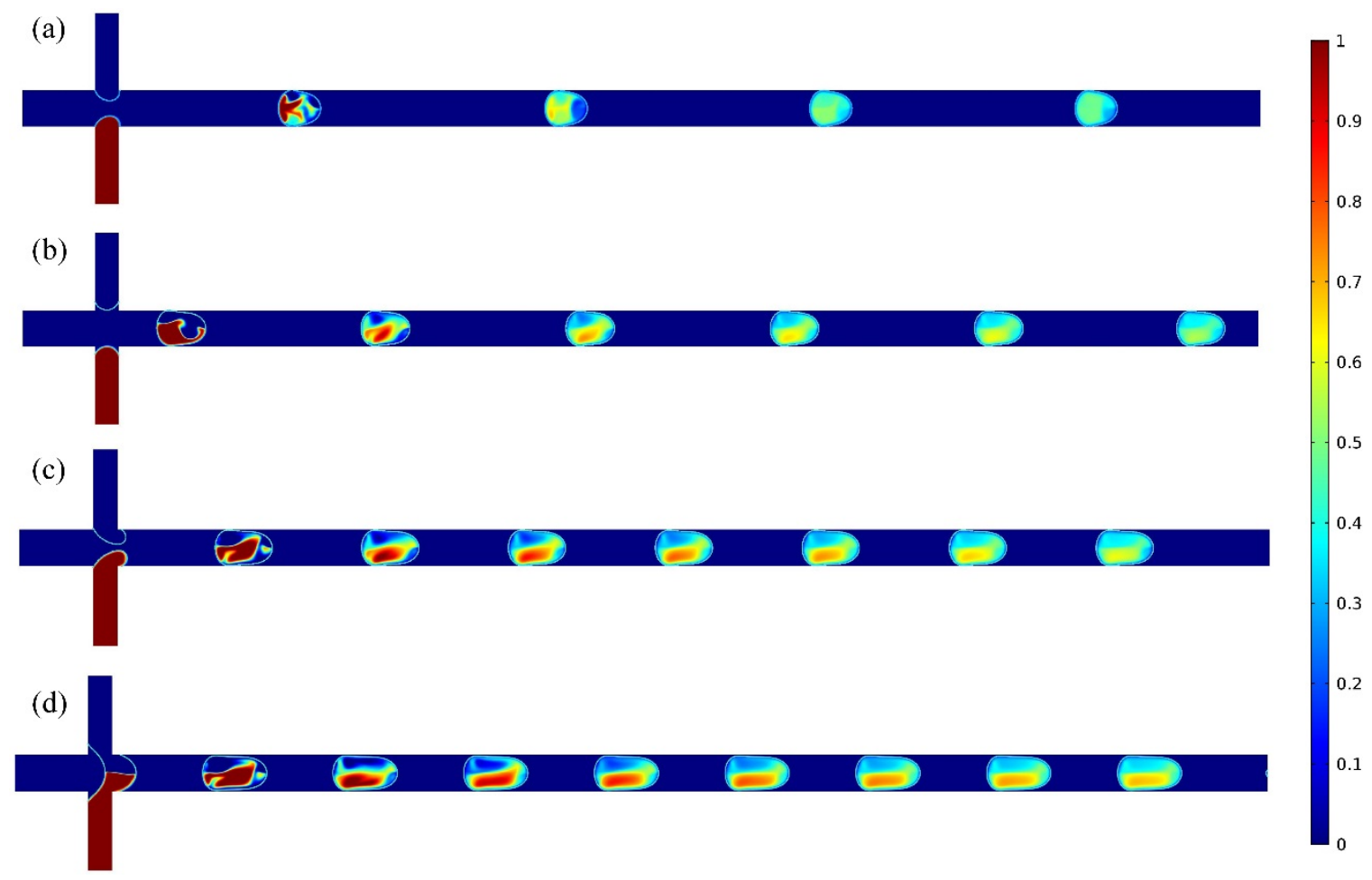

590 FIG. 14 Nanoparticles concentration distribution in microdroplets of different sizes (a) $Q_{r}=6.67$ 591 , (b) $Q_{r}=4$, (c) $Q_{r}=2$ and (d) $Q_{r}=1.33\left(\mathbf{B}=60 \mathrm{mT}, Q_{c}=0.12 \mathrm{ml} / \mathrm{h}, 0.018 \leq Q_{d} \leq 0.09 \mathrm{ml} / \mathrm{h}\right.$ 592 ).

593

594

595 


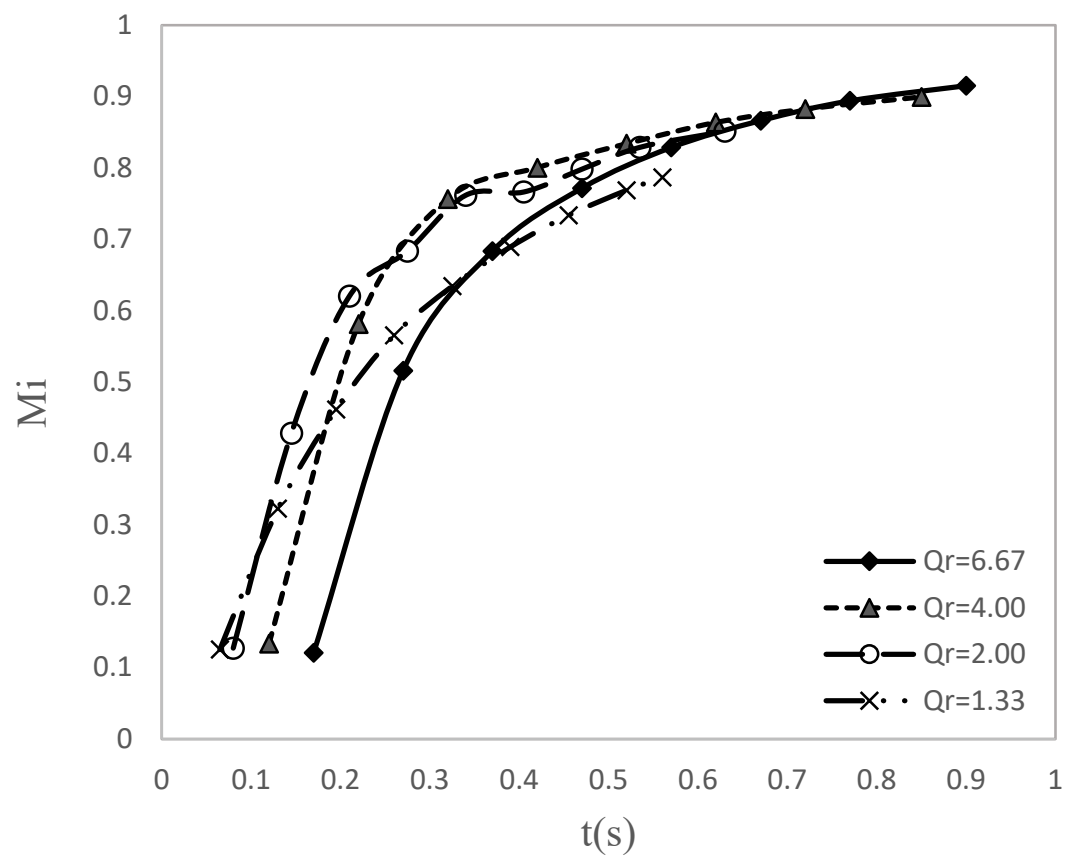

597 Fig. 15 Mixing efficiency in droplet versus time for different values of the droplet sizes $\left.598 \quad \mathbf{B}=60 \mathrm{mT}, Q_{c}=0.12 \mathrm{ml} / \mathrm{h}, 0.018 \leq Q_{d} \leq 0.09 \mathrm{ml} / \mathrm{h}\right)$.

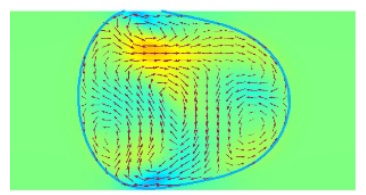

$Q_{r}=6.67$

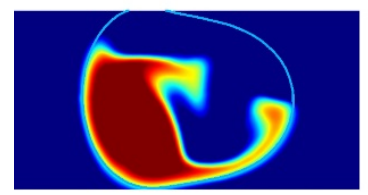

$Q_{r}=6.67$

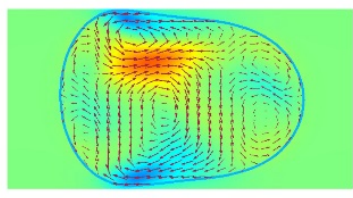

$Q_{r}=4.00$

(a)

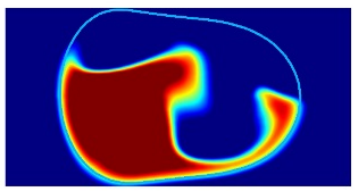

$Q_{r}=4.00$

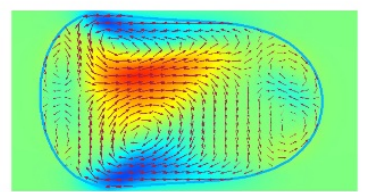

$Q_{r}=2.00$

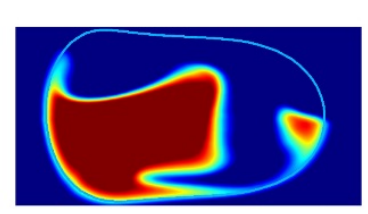

$Q_{r}=2.00$
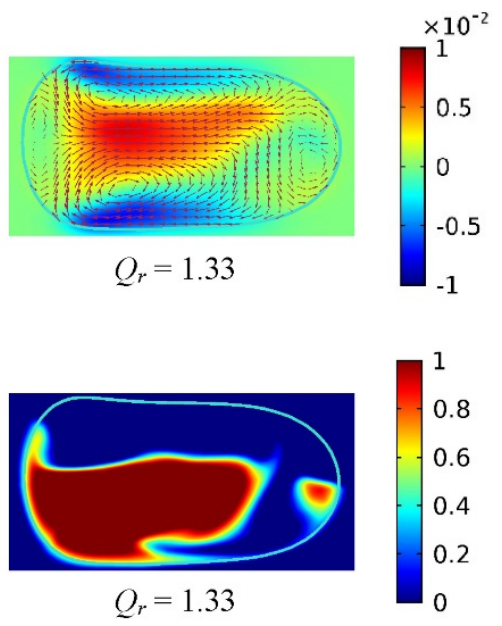

(b)

600

601

602

603

FIG. 16 Mixing performance of droplets with various sizes and 300 micrometers center to center distance from the cross junction, (a) relative velocities and streamlines in the droplet frame of the reference, (b) nanoparticles concentration distribution inside the droplets $(\mathbf{B}=60 \mathrm{mT}$, $\left.Q_{c}=0.12 \mathrm{ml} / \mathrm{h}, 0.018 \leq Q_{d} \leq 0.09 \mathrm{ml} / \mathrm{h}\right)$. 


\section{Evaluation of mixing in a combined diagram}

606 For more insight into the mixing behavior inside the droplets, three different parameters were 607 employed as indicators for droplet velocity, droplet size and the magnetic field strength:

608

609

610

$L s_{-} a v=\left(L_{d}-L_{d_{-} \min }\right) /\left(L_{d_{-} \max }-L_{d_{-} \min }\right)$

$C a_{-} a v=\left(C a_{d}-C a_{d_{-} \min }\right) /\left(C a_{d_{-} \max }-C a_{d_{-} \min }\right)$

611

$B m_{-} a v=\left(B_{m}-B_{m_{-} \min }\right) /\left(B_{m_{-} \max }-B_{m_{-} \min }\right)$

612 where $L_{d}$ is the droplet length within the channel, $C a_{d}$ is the dispersed phase Capillary number

$613 B_{m}$ is the magnetic Bond number and max and min stand for the maximum and minimum value

614 of the related parameters in a group of studied items (cases). The reason for the definition of the 615 parameters based on their maximum and minimum values is obtaining a unit range of variations 616 (between zero and one) which is a convenient way for showing all these parameters on a diagram.

617 The $C a_{d}$ and $B_{m}$ can be calculated by:

$618 C a_{d}=\bar{\eta}_{d} U_{d} / \sigma$

$619 B_{m}=\mu_{0} R_{0} H^{2} / \sigma$

620 where $\bar{\eta}_{d}=3.75 \mathrm{mPa} \cdot \mathrm{s}$ is the average viscosity inside the droplet, $U_{d}$ is the droplet velocity and

$621 R_{0}$ is the droplet radius for without deformation state.

622 Fig. 17 represents the combined diagram, which evaluates the variation of the mixing index with

623 regard to the three parameters ( $\left.L s_{-} a v, C a_{-} a v, B m_{-} a v\right)$, concurrently. According to this

624 figure, for items 1 to 5 where $L s_{-} a v$ and $C a_{-} a v$ are almost constant, there is a specific value 625 for $B m_{-} a v$ where the mixing index is maximized (item 4). For items 6 to 10, Bm_av is nearly 
626 fixed, $L s_{-} a v$ changes smoothly and $\mathrm{Ca} a_{-} a v$ shifts significantly. Also for this case, the value of 627 MI peakes where the ratio of the $\mathrm{Bm}_{-} a v$ to $\mathrm{Ca}_{-} a v$ is a certain value (item 7). The comparison 628 of two items 4 and 7 illustrates that higher $C a_{-} a v$ and relatively smaller $L s_{-} a v$ can result in 629 larger mixing efficiency. Verifying items 10 to 14 shows the worsening of the mixing with striking 630 growth of $\mathrm{Ls}_{-} a v$ and $\mathrm{Ca}_{-} a v$, while $\mathrm{Bm}_{-} a v$ is near constant. These observations support our 631 previous discussion that optimized mixing occurs for a given ratio of the magnetic force to the 632 shear force. And using smaller drops has a positive effect in mixing enhancement. Also based on 633 the diagram synchronized increase of the magnetic and the shear forces improves mixing.

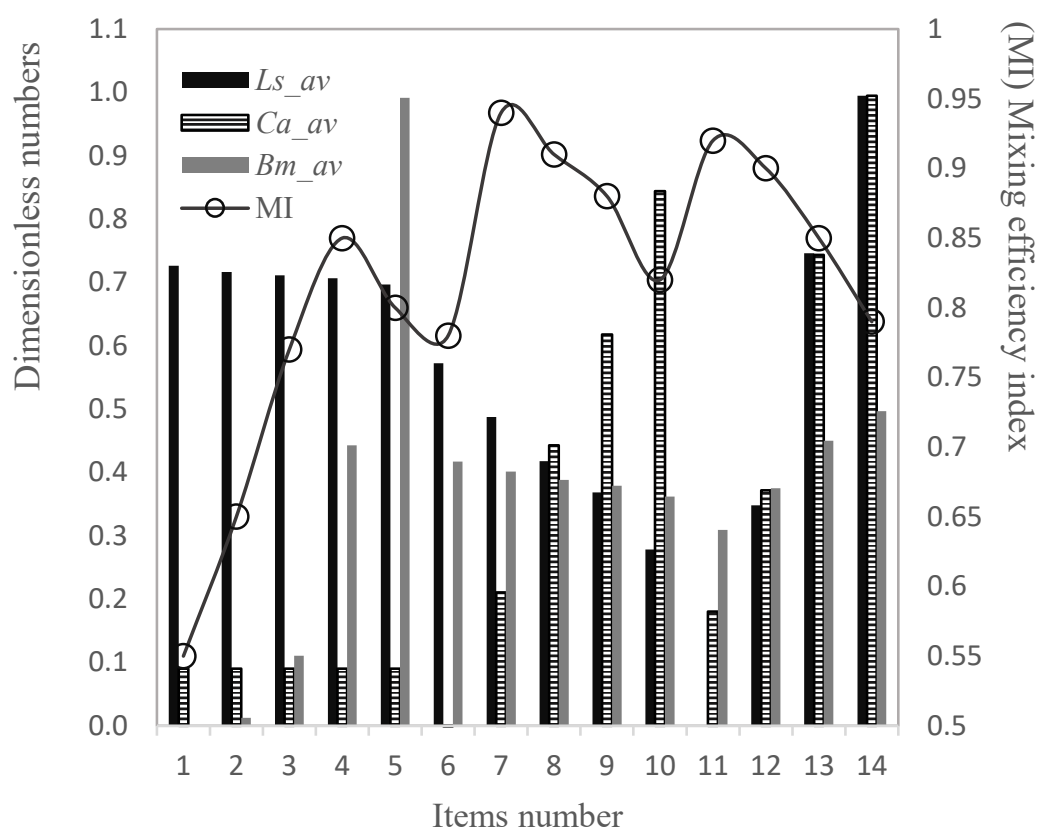

635

636 FIG. 17 Combined diagram for simultaneous evaluation of mixing under influence of different 637 parameters 
639 This work demonstrates active mixing of ferrofluid with a diamagnetic glycerol-water mixture in 640 microdroplets under a uniform magnetic field. Low Reynolds and Capillary numbers provide 641 laminar flow and merging droplet formation regimes, respectively, while high Peclet number 642 offers negligible diffusion. Uniform magnetic field induces a magnetic force on both ferrofluid-oil 643 and ferrofluid-diamagnetic mixture interfaces, where mismatched magnetic susceptibility exists.

644 The magnetic force exerting on the droplet boundary leads to droplet deformation, and that 645 applying on miscible phases interface destroys the symmetry of the flow patterns in the droplet.

646 The influence of three factors including, magnetic force, droplet velocity and droplet size on 647 mixing efficiency was examined. For the first factor, results showed that a strong magnetic force 648 rapidly redistributes particles and accumulates them in the middle and rear vortices. However, 649 under a low magnetic field strength, the perturbations caused by magnetic force are very poor, and 650 subsequently, particles transportation between two halves of the droplet occurs mainly through the 651 diffusion. Additionally, it was seen that the increase of droplet velocity and size have a negative 652 impact on the response of the particles to the magnetic field as it reinforces shear forces and 653 vortices strength inside the droplet. Particles convection inside the droplet is regulated by the 654 interaction between the magnetic force and shear force that proceed from the droplet movement 655 within the channel. Our results indicated that to attain optimized mixing a balance between the 656 magnetic force and shear force should be realized. The dominant magnetic force vigorously 657 manipulates vortices and in a very short time homogenizes particles in line with the magnetic field 658 at droplet flanks and rear part while droplet tip becomes nearly empty from particles. Then vortices 659 evolve to their symmetrical configuration, and mass transport to the droplet tip mostly occurs 660 through the very slow mechanism of diffusion. On the other hand, powerful shear force excludes 661 magnetic force from developing inhomogeneity in the recirculation zones. In this case, the 
662

663

664

665

666

667

668

669

670

671

672

673

674

675 676 mixing in the microchannel.

magnetic field is only able to drag a minor part of particles toward the weaker vortices in the droplet tip or boundaries between vortices and significant amounts of particles remain in the lower half of the droplet. At intermediate levels, gentle changes in vortices by the magnetic field, Spreads the particles uniformly across the droplet. The mixing efficiency of $94 \%$ was demonstrated for this condition. Also, it was observed that smaller droplets are better candidates for droplet mixing. Hence, the thinner diffusion barriers between their vortices make the mass exchange between inner vortices easier. From a practical point of view, controlling the mixing with adjustment of the flowrates through syringe pumps or regulating the magnetic field through a customized electromagnet is more affordable than other approaches. Nevertheless, other strategies for controlling mixing are possible. Employing ferrofluids with different properties (nanoparticles concentration, magnetic susceptibility, etc.) could be useful in tuning the magnetic force and flow characteristics. Finally, to speed up the mixing process, one can concurrently increase the magnetic force and the droplet speed until the formation regime changes to alternating one, in which different kinds of magnetic configuration can be employed to accomplish droplet merging and

\section{Acknowledgements}

678 N. T. N. acknowledges funding support from Australian Research Council, grant number 679 DP180100055.

\section{Reference}

1. S.-Y. Teh, R. Lin, L.-H. Hung, andA. P. Lee, "Droplet microfluidics," Lab on a Chip 8, 198 (2008).

684 3. Y. Zhu, andQ. Fang, "Analytical detection techniques for droplet microfluidics-A review," Analytica 685 Chimica Acta 787, 24 (2013).

686 4. Z. Zhang, J. Xu, C. J. M. Drapaca, andNanofluidics, "Particle squeezing in narrow confinements," 22, 687120 (2018). 
5. P. Neužil, S. Giselbrecht, K. Länge, T. J. Huang, andA. Manz, "Revisiting lab-on-a-chip technology for drug discovery," Nature Reviews Drug Discovery 11, 620 (2012).

6. C. Chawaree, P. Anna, D. Hansi, P. Pascal, B. Vladimir, andG. Andrew D., "Single-Virus Droplet Microfluidics for High-Throughput Screening of Neutralizing Epitopes on HIV Particles," Cell Chemical Biology 24, 751 (2017).

7. E. Z. Macosko, A. Basu, R. Satija, J. Nemesh, K. Shekhar, M. Goldman, I. Tirosh, A. R. Bialas, N. Kamitaki, E. M. Martersteck, J. J. Trombetta, D. A. Weitz, J. R. Sanes, A. K. Shalek, A. Regev, andS. A. McCarroll, "Highly Parallel Genome-wide Expression Profiling of Individual Cells Using Nanoliter Droplets," Cell 161, 1202 (2015).

8. N. Kashaninejad, M. J. A. Shiddiky, andN.-T. Nguyen, "Advances in Microfluidics-Based Assisted Reproductive Technology: From Sperm Sorter to Reproductive System-on-a-Chip," 2, 1700197 (2018). 9. D. Ferraro, J. Champ, B. Teste, M. Serra, L. Malaquin, J.-L. Viovy, P. de Cremoux, andS. Descroix, "Microfluidic platform combining droplets and magnetic tweezers: application to HER2 expression in cancer diagnosis," Scientific Reports 6, 25540 (2016).

10. X. Chen, andCarolyn L. Ren, "A microfluidic chip integrated with droplet generation, pairing, trapping, merging, mixing and releasing," RSC Advances 7, 16738 (2017).

11. E. Amstad, M. Chemama, M. Eggersdorfer, L. R. Arriaga, M. P. Brenner, andD. A. Weitz, "Robust scalable high throughput production of monodisperse drops," Lab Chip 16, 4163 (2016).

12. T. Glawdel, C. Elbuken, andC. Ren, "Passive droplet trafficking at microfluidic junctions under geometric and flow asymmetries," Lab on a Chip 11, 3774 (2011).

13. K. Zhao, andD. Li, "Tunable Droplet Manipulation and Characterization by ac-DEP," ACS applied materials \& interfaces 10, 36572 (2018).

14. RuihuaDing, W. L. Ung, J. A. Heyman, andD. A. Weitz, "Sensitive and predictable separation of microfluidic droplets by size using in-line passive filter," 11, 014114 (2017).

15. H. Geng, J. Feng, L. M. Stabryla, andS. K. Cho, "Dielectrowetting manipulation for digital microfluidics: creating, transporting, splitting, and merging of droplets," Lab on a Chip 17, 1060 (2017). 16. M.-A. Nguyen, B. Srijanto, C. P. Collier, S. T. Retterer, andS. A. Sarles, "Hydrodynamic trapping for rapid assembly and in situ electrical characterization of droplet interface bilayer arrays," Lab on a Chip 16, 3576 (2016).

17. G. Yesiloz, M. S. Boybay, andC. L. Ren, "Effective Thermo-Capillary Mixing in Droplet Microfluidics Integrated with a Microwave Heater," Analytical Chemistry 89, 1978 (2017). 18. E. Samiei, M. D. de Leon Derby, A. V. den Berg, andM. Hoorfar, "An electrohydrodynamic technique for rapid mixing in stationary droplets on digital microfluidic platforms," Lab Chip 17, 227 (2017).

19. J. S. Sander, R. M. Erb, C. Denier, andA. R. Studart, "Magnetic Transport, Mixing and Release of Cargo with Tailored Nanoliter Droplets," 24, 2582 (2012).

20. D. Jiang, S. Lee, S. W. Bae, andS. Y. Park, "Smartphone integrated optoelectrowetting (SiOEW) for onchip sample processing and microscopic detection of water quality," Lab Chip 18, 532 (2018).

21. Q. Liu, andB. Xu, "Actuating Water Droplets on Graphene via Surface Wettability Gradients," Langmuir 31, 9070 (2015).

22. J. K. Park, andS. Kim, "Droplet manipulation on a structured shape memory polymer surface," Lab on a Chip 17, 1793 (2017).

23. C. Bakli, S. H. P. D, andS. Chakraborty, "Mimicking wettability alterations using temperature gradients for water nanodroplets," Nanoscale 9, 12509 (2017).

24. A. Munaz, H. Kamble, M. J. A. Shiddiky, andN.-T. Nguyen, "Magnetofluidic micromixer based on a complex rotating magnetic field," RSC Advances 7, 52465 (2017).

25. T. Q. Bui, S. N.-C. Ton, A. T. Duong, andH. T. Tran, "Size-dependent magnetic responsiveness of magnetite nanoparticles synthesised by co-precipitation and solvothermal methods," Journal of Science: Advanced Materials and Devices 3, 107 (2018). 
26. W. H. Chong, Y. Huang, T. N. Wong, K. T. Ooi, andG.-P. Zhu, "Magnetic Nanorobots, Generating Vortexes Inside Nanoliter Droplets for Effective Mixing," 3, 1700312 (2018). 27. Y. Fu, L. Bai, S. Zhao, X. Zhang, Y. Jin, andY. Cheng, "Simulation of reactive mixing behaviors inside micro-droplets by a lattice Boltzmann method," Chemical Engineering Science 181, 79 (2018). 28. J. Wang, J. Wang, L. Feng, andT. Lin, "Fluid mixing in droplet-based microfluidics with a serpentine microchannel," RSC Advances 5, 104138 (2015).

29. H. Yu, T.-B. Nguyen, S. H. Ng, andT. Tran, "Mixing control by frequency variable magnetic micropillar," RSC Advances 6, 11822 (2016).

30. N.-T. Nguyen, "Micro-magnetofluidics: interactions between magnetism and fluid flow on the microscale," Microfluidics and Nanofluidics 12, 1 (2012).

31. Y. Zhang, andT. H. Wang, "Full-range magnetic manipulation of droplets via surface energy traps enables complex bioassays," Advanced materials (Deerfield Beach, Fla.) 25, 2903 (2013).

32. T. Roy, A. Sinha, S. Chakraborty, R. Ganguly, andl. K. Puri, "Magnetic microsphere-based mixers for microdroplets," 21, 027101 (2009).

33. V. B. Varma, A. Ray, Z. M. Wang, Z. P. Wang, andR. V. Ramanujan, "Droplet Merging on a Lab-on-aChip Platform by Uniform Magnetic Fields," Scientific Reports 6, 37671 (2016).

34. E. Olsson, andG. Kreiss, "A conservative level set method for two phase flow," Journal of Computational Physics 210, 225 (2005).

35. G.-P. Zhu, andN.-T. Nguyen, "Rapid magnetofluidic mixing in a uniform magnetic field," Lab on a Chip 12, 4772 (2012).

36. E. Y. Kenig, A. A. Ganguli, T. Atmakidis, andP. Chasanis, "A novel method to capture mass transfer phenomena at free fluid-fluid interfaces," Chemical Engineering and Processing: Process Intensification 50, 68 (2011).

37. M. J. Rivera, M. Trujillo, V. Romero-García, J. A. López Molina, andE. Berjano, "Numerical resolution of the hyperbolic heat equation using smoothed mathematical functions instead of Heaviside and Dirac delta distributions," International Communications in Heat and Mass Transfer 46, 7 (2013).

38. Q. A. Pankhurst, J. Connolly, S. K. Jones, andJ. Dobson, "Applications of magnetic nanoparticles in biomedicine," Journal of Physics D: Applied Physics 36, R167 (2003).

39. O. Lavrova, G. Matthies, T. Mitkova, V. Polevikov, andL. Tobiska, "Numerical treatment of free surface problems in ferrohydrodynamics," Journal of Physics: Condensed Matter 18, S2657 (2006). 40. J. Liu, Y. F. Yap, andN.-T. Nguyen, "Numerical study of the formation process of ferrofluid droplets," 23, 072008 (2011).

41. B. I. Kharisov, H. V. R. Dias, O. V. Kharissova, A. Vázquez, Y. Peña, andl. Gómez, "Solubilization, dispersion and stabilization of magnetic nanoparticles in water and non-aqueous solvents: recent trends," RSC Advances 4, 45354 (2014).

42. L. Yang, S. Li, J. Liu, andJ. Cheng, "Fluid mixing in droplet-based microfluidics with T junction and convergent-divergent sinusoidal microchannels," Electrophoresis 39, 512 (2018).

43. J. R. Cash, "Modified extended backward differentiation formulae for the numerical solution of stiff initial value problems in ODEs and DAEs," Journal of Computational and Applied Mathematics 125, 117 (2000).

44. B. Stute, V. Krupp, andE. von Lieres, "Performance of iterative equation solvers for mass transfer problems in three-dimensional sphere packings in COMSOL," Simulation Modelling Practice and Theory 33, 115 (2013).

45. G. Nicholas I. M., H. Yifan, andS. Jennifer A., A numerical evaluation of sparse direct solvers for the solution of large sparse, symmetric linear systems of equations Document No. Number, 2005.

46. W. Frei, Keeping Track of Element Order in Multiphysics Models (2016). 
47. J. Jouya, Z. Markus, L. Nils, W. Ola, andB. Karl, Optimization of Artificial Diffusion Stabilization

783 Techniques and Corresponding Mesh density Distribution in Drift Dominated Transport of Diluted Species 784 (BOSTON, 2012).

785 48. H. Shu-Ren, "Numerical Simulation of Immiscible Fluids with FEM Level Set Techniques," Doktors der 786 Naturwissenschaften Universit"at Dortmund, 2007.

787 49. M. Schmitt, andH. Stark, "Marangoni flow at droplet interfaces: Three-dimensional solution and 788 applications," 28, 012106 (2016).

789 50. A. Riaud, H. Zhang, X. Wang, K. Wang, andG. Luo, "Numerical Study of Surfactant Dynamics during 790 Emulsification in a T-Junction Microchannel," Langmuir 34, 4980 (2018).

791 51. B. Zheng, J. D. Tice, andR. F. Ismagilov, "Formation of Droplets of Alternating Composition in 792 Microfluidic Channels and Applications to Indexing of Concentrations in Droplet-Based Assays," 793 Analytical Chemistry 76, 4977 (2004).

794 52. I.-L. Ngo, T.-D. Dang, C. Byon, andS. W. Joo, "A numerical study on the dynamics of droplet formation 795 in a microfluidic double T-junction," Biomicrofluidics 9, 024107 (2015).

796 53. Z. Zhang, C. Drapaca, D. Gritsenko, andJ. Xu, "Pressure of a viscous droplet squeezing through a short 797 circular constriction: An analytical model," 30, 102004 (2018).

798 54. Z. Zhang, C. Drapaca, X. Chen, andJ. Xu, "Droplet squeezing through a narrow constriction: Minimum 799 impulse and critical velocity," 29, 072102 (2017).

800 55. C. M. Leong, Y. Gai, andS. K. Y. Tang, "Internal flow in droplets within a concentrated emulsion

801 flowing in a microchannel," 28, 112001 (2016).

802 56. M. Hein, M. Moskopp, andR. Seemann, "Flow field induced particle accumulation inside droplets in 803 rectangular channels," Lab Chip 15, 2879 (2015).

804 57. S. Ma, J. M. Sherwood, W. T. S. Huck, andS. Balabani, "On the flow topology inside droplets moving 805 in rectangular microchannels," Lab on a Chip 14, 3611 (2014). 